\title{
Mesenchymal Bmp3b expression maintains skeletal muscle integrity and decreases in age-related sarcopenia
}

\author{
Akiyoshi Uezumi, ${ }^{1}$ Madoka Ikemoto-Uezumi, ${ }^{1}$ Heying Zhou, ${ }^{1}$ Tamaki Kurosawa, ${ }^{1}$ Yuki Yoshimoto, ${ }^{1}$ Masashi Nakatani, ${ }^{2}$ \\ Keisuke Hitachi, ${ }^{3}$ Hisateru Yamaguchi, ${ }^{4}$ Shuji Wakatsuki, ${ }^{5}$ Toshiyuki Araki, ${ }^{5}$ Mitsuhiro Morita, ${ }^{6}$ Harumoto Yamada, ${ }^{6}$ \\ Masashi Toyoda, ${ }^{7}$ Nobuo Kanazawa, ${ }^{8}$ Tatsu Nakazawa, ${ }^{9}$ Jun Hino, ${ }^{10}$ So-ichiro Fukada, ${ }^{11}$ and Kunihiro Tsuchida ${ }^{3}$ \\ 1Muscle Aging and Regenerative Medicine, Tokyo Metropolitan Institute of Gerontology (TMIG), Tokyo, Japan. ${ }^{2}$ Faculty of Rehabilitation and Care, Seijoh University, Tokai, Japan. ${ }^{3}$ Division for Therapies \\ against Intractable Diseases, Institute for Comprehensive Medical Science (ICMS), Fujita Health University, Toyoake, Japan. ${ }^{4}$ Department of Medical Technology, School of Nursing and Medical Care, Yokkaichi \\ Nursing and Medical Care University, Yokkaichi, Japan. ${ }^{5}$ Department of Peripheral Nervous System Research, National Institute of Neuroscience, National Center of Neurology and Psychiatry, Tokyo, Japan. \\ ${ }^{6}$ Department of Orthopaedic Surgery, Fujita Health University, Toyoake, Japan. ${ }^{7}$ Vascular Medicine, TMIG, Tokyo, Japan. ${ }^{8}$ Department of Surgery, Tokyo Metropolitan Geriatric Hospital and Institute of \\ Gerontology (TMCHIG), Tokyo, Japan. ${ }^{9}$ Seibo Hospital, Tokyo, Japan. ${ }^{10}$ Department of Biochemistry, National Cerebral and Cardiovascular Center Research Institute, Osaka, Japan. ${ }^{11 P r o j e c t ~ f o r ~ M u s c l e ~ S t e m ~}$ \\ Cell Biology, Graduate School of Pharmaceutical Sciences, Osaka University, Osaka, Japan.
}

\begin{abstract}
Age-related sarcopenia constitutes an important health problem associated with adverse outcomes. Sarcopenia is closely associated with fat infiltration in muscle, which is attributable to interstitial mesenchymal progenitors. Mesenchymal progenitors are nonmyogenic in nature but are required for homeostatic muscle maintenance. However, the underlying mechanism of mesenchymal progenitor-dependent muscle maintenance is not clear, nor is the precise role of mesenchymal progenitors in sarcopenia. Here, we show that mice genetically engineered to specifically deplete mesenchymal progenitors exhibited phenotypes markedly similar to sarcopenia, including muscle weakness, myofiber atrophy, alterations of fiber types, and denervation at neuromuscular junctions. Through searching for genes responsible for mesenchymal progenitor-dependent muscle maintenance, we found that $B m p 3 b$ is specifically expressed in mesenchymal progenitors, whereas its expression level is significantly decreased during aging or adipogenic differentiation. The functional importance of BMP3B in maintaining myofiber mass as well as muscle-nerve interaction was demonstrated using knockout mice and cultured cells treated with BMP3B. Furthermore, the administration of recombinant BMP3B in aged mice reversed their sarcopenic phenotypes. These results reveal previously unrecognized mechanisms by which the mesenchymal progenitors ensure muscle integrity and suggest that age-related changes in mesenchymal progenitors have a considerable impact on the development of sarcopenia.
\end{abstract}

\section{Introduction}

Sarcopenia, the loss of skeletal muscle mass and strength, constitutes an important health problem associated with adverse outcomes such as disability, poor quality of life, and even death. A decline in muscle strength precedes the loss of muscle mass in older adults (1), suggesting decreased muscle quality as a causal factor of sarcopenia. Fat infiltration is a notable change that affects muscle quality with advancing age. Older and frail individuals demonstrate increased fat infiltration in the muscles (2, 3 ), and high levels of fat infiltration impair the adaptive response to exercise training among the elderly (4). Ectopic fat formation in muscle is attributable to nonmyogenic mesenchymal progenitors, also known as fibrogenic/adipogenic progenitors $(5,6)$. These progenitors reside in the muscle interstitial space and specifically express PDGFR $\alpha$ (5). Mesenchymal progenitors do not differentiate into myogenic cells but exhibit the potential to dif-

Conflict of interest: The authors have declared that no conflict of interest exists. Copyright: () 2021, American Society for Clinical Investigation.

Submitted: April 27, 2020; Accepted: October 29, 2020; Published: January 4, 2021.

Reference information: J Clin Invest. 2021;131(1):e139617.

https://doi.org/10.1172/JCl139617. ferentiate into other mesenchymal lineages, and they have also been demonstrated to be the origin of fibrosis $(7,8)$ and heterotopic ossification $(9,10)$ in muscle.

In addition to their roles in the development of disease pathology in the muscles, mesenchymal progenitors play an important role in muscle regeneration. Regeneration of adult myofibers depends entirely on muscle stem cells called satellite cells. Satellite cells are located between the basal lamina and plasma membrane of myofibers, and therefore, differ from the mesenchymal progenitors that reside in the interstitium. Genetically engineered mice with conditional depletion of satellite cells demonstrate a complete loss of their regenerative ability (11-13), indicating that satellite cells are essential for muscle regeneration in adults and cannot be compensated by the functions of other cell types. Transcription factor TCF4, also known as transcription factor 7-like 2, is expressed in muscle interstitial cells, including PDGFR $\alpha^{+}$mesenchymal progenitors. Murphy et al. reported that the depletion of $\mathrm{TCF}^{+}$cells during muscle regeneration results in altered satellite cell dynamics, premature satellite cell differentiation, depletion of the early pool of satellite cells, and insufficient myofiber regeneration (12). A recent report also demonstrated the indispensability of mesenchymal 
A

Pdgfra-CreERT $\left(P_{\alpha}-C E\right)$

Pdgfra promoter CreER $^{\top} \rightarrow \times$ Rosa26 locus

B
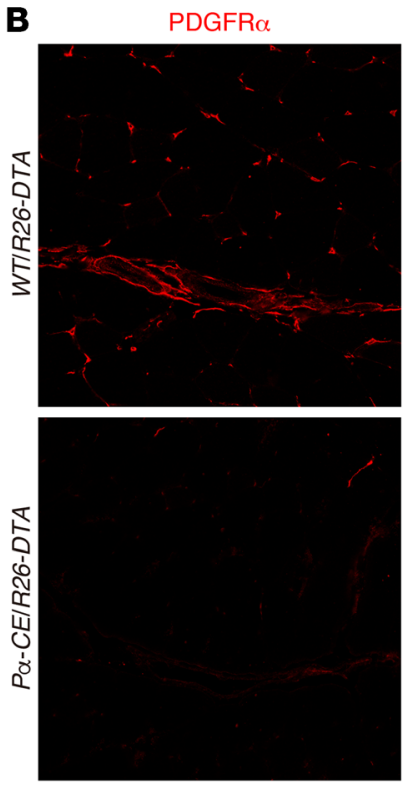

$\mathbf{E}$

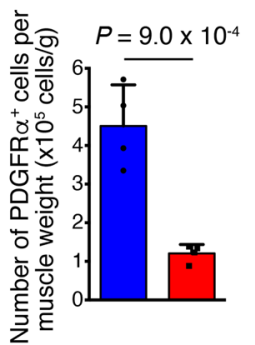

PDGFR $\propto$ Dystrophin DAPI
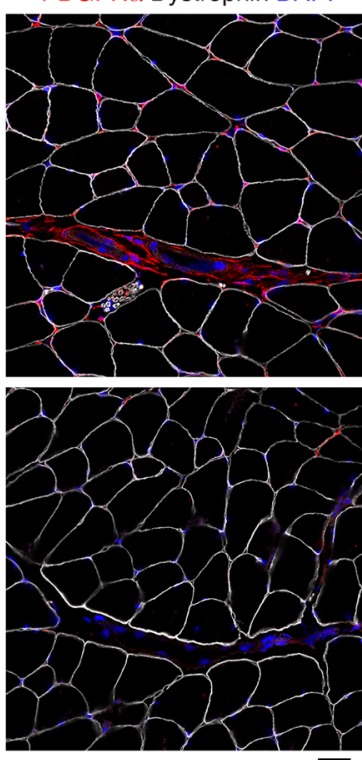

C

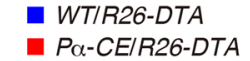

$2 \mathrm{~d}$

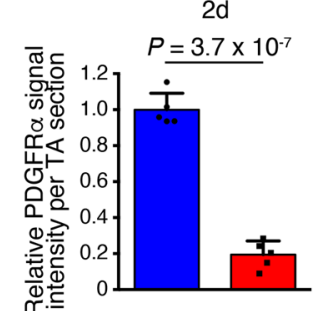

$6 \mathrm{w}$

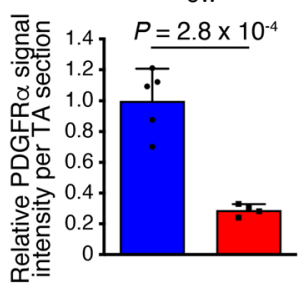

WTIR26-DTA

- $P \alpha-C E / R 26-D T A$

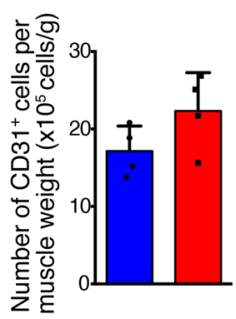

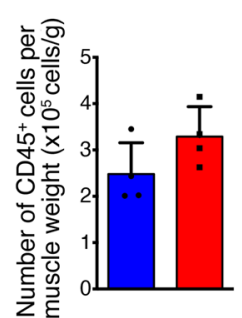

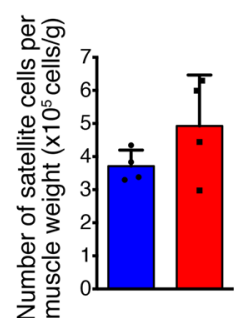

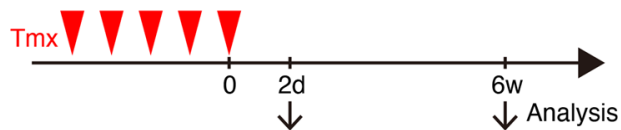

D
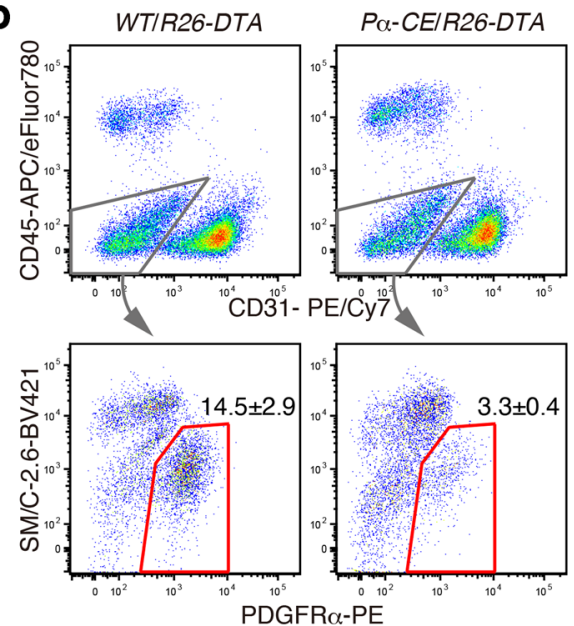

Figure 1. Specific and efficient depletion of mesenchymal progenitors. (A) Scheme of mesenchymal progenitor depletion. (B) Immunostaining of tibialis anterior (TA) muscle for PDGFR $\alpha$ and dystrophin. (C) Quantification of PDCFR $\alpha$ signal intensity. $n=5$ at 2 days of $\operatorname{Tmx}, n=5$ for WT/R26DTA and $n=4$ for $P \alpha$-CE/R26-DTA at 6 weeks of Tmx. (D) FACS analysis of hind limb muscles. Red polygonal region indicates PDGFR $\alpha^{+}$mesenchymal progenitors. The percentage of PDCFR $\alpha^{+}$cells in the total mononucleated cells is shown. $n=4$. (E) Quantification of cell number per muscle weight. $n=4$. Data represent the mean $\pm S D$; 2 -sided unpaired $t$ test (C and $\mathbf{E}$ ). Scale bar: $20 \mu \mathrm{m}$ (B). progenitors in muscle regeneration by conditional depletion of PDGFR $\alpha^{+}$cells (14). Thus, mesenchymal progenitors play a supportive role by regulating satellite cell behavior. Hence, they are required for efficient and sufficient muscle regeneration.

Several lines of evidence indicate that regular regeneration is not required for the maintenance of homeostasis in normal uninjured myofibers during the process of natural aging (15). There is little evidence of ongoing myonecrosis and subsequent myogenesis in most people, even those who regularly perform mild exercise (16). This notion is strongly supported by studies conducted with satellite cell-ablated aged mice, in which most muscles demonstrate normal phenotype with no exacerbation of sarcopenia $(17,18)$. In contrast, a recent study showed that mice with depleted mesenchymal progenitors exhibit muscle atrophy under steady-state conditions (14). Therefore, it is becoming clearer that mesenchymal progenitors also play an essential role in homeostatic muscle maintenance. However, the mechanism by which mesenchymal progenitors maintain muscle integrity is not clear, nor is the precise role of these cells in muscle aging.
In this study, through searching for the genes responsible for mesenchymal progenitor-dependent muscle maintenance, we found that $B m p 3 b$ is specifically expressed in mesenchymal progenitors and its expression is significantly decreased during aging or adipogenic differentiation. We demonstrated the functional importance of BMP3B in both in vivo and in vitro experiments. Furthermore, the administration of BMP3B to aged mice resulted in improved energy metabolism and an increase in muscle mass and strength. Our results demonstrate underlying mechanisms of the maintenance of muscle health by interstitial mesenchymal progenitors and provide a mechanistic insight into how mesenchymal progenitors contribute to sarcopenia.

\section{Results}

Depletion of mesenchymal progenitors leads to muscle weakness and atrophy. To elucidate the role of mesenchymal progenitors under steady-state conditions, we utilized Pdgfra-CreER (hereafter referred to as $P \alpha-C E$ ) mice (19). Crossing these animals with R26-EYFP mice followed by tamoxifen (Tmx) administration confirmed the highly specific recombination in PDGFR $\alpha^{+}$cells (PDG- 
A

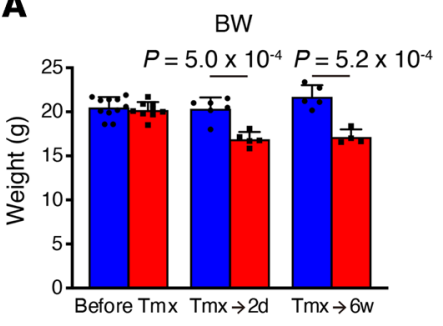

\section{B}

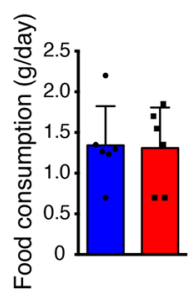

C $\operatorname{Tm} x \rightarrow 2 \mathrm{~d} \quad \operatorname{Tm} x \rightarrow 6 \mathrm{w}$ Grip strength Grip strength

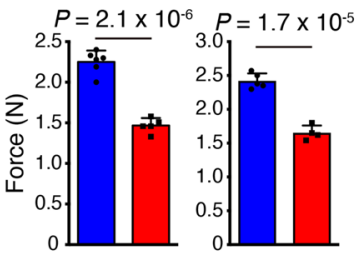

D

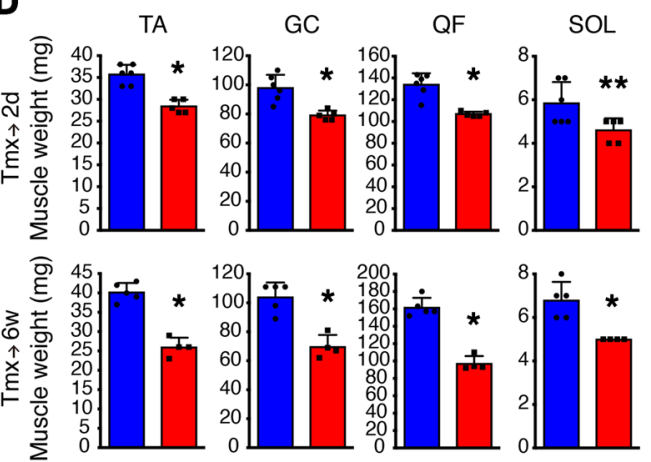

E

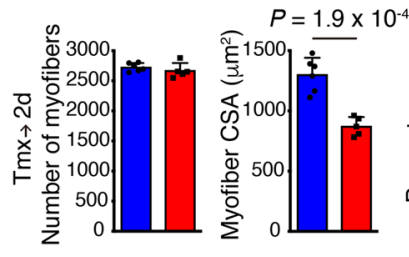

$P=3.1 \times 10^{-3} \quad$ Myofiber CSA $\left(\mu \mathrm{m}^{2}\right)$
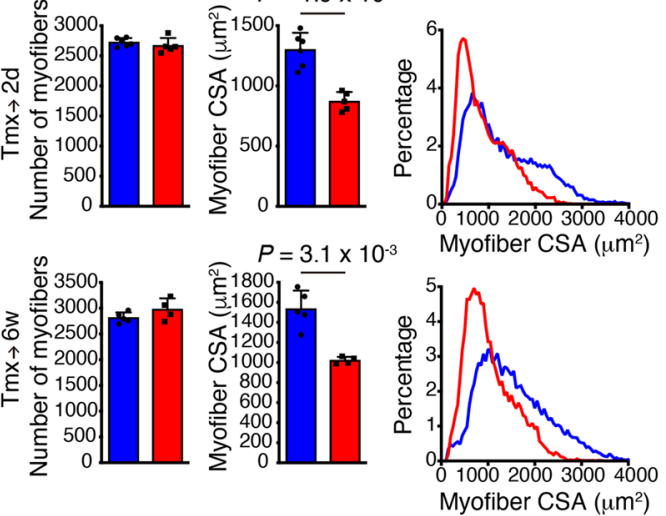
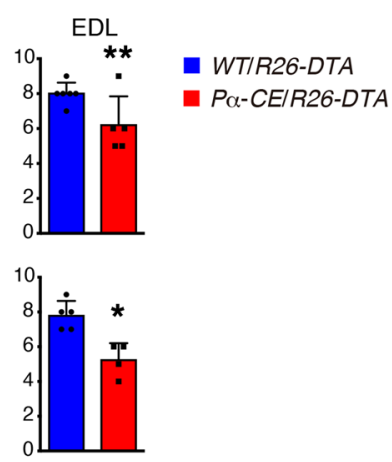

RF

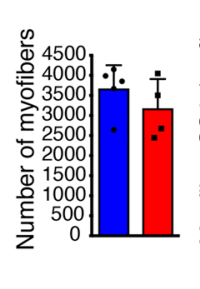

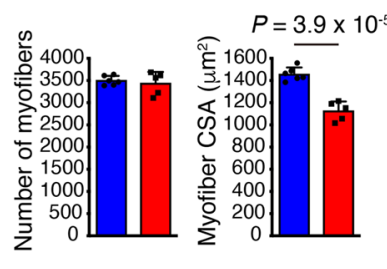

$P=\underline{1.2 \times 10^{-3}}$

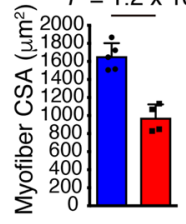

Figure 2. Mesenchymal progenitor depletion leads to muscle weakness and atrophy. (A-E) Body weight (A), food intake during the last 3 days of Tmx injection (B), grip strength (C), muscle weight (D), and number, CSA, and CSA distribution of myofibers in TA and RF muscles (E). $n=11,6,5$ for WT/R26-DTA and $n=8,5,4$ for $P \alpha$-CE/R26-DTA prior to Tmx and at 2 days of Tmx and 6 weeks of Tmx. $n=6$ for food intake. TA, tibialis anterior; GC, gastrocnemius; QF, quadriceps femoris; SOL, soleus $E D L$, extensor digitorum longus; RF, rectus femoris. (F) Expression of $F b x 032$ in the TA muscle. $n=6$ for WT/R26-DTA and $n=5$ for $P \alpha$-CE/R26-DTA.

(C) Scheme of the reconstitution of mesenchymal progenitors. Contralateral TA was used as a control with PBS injection. Fluorescent dye (DiD) was used to visualize the PBS-distributed area in the final injection. (H) Fluorescence images of GFP, DiD, PDGFR $\alpha$, and laminin $\alpha 2$. (I) Muscle weight and CSA of mesenchymal progenitor-reconstituted mice. $n=$ 4. Data represent the mean $\pm S D ; 2$-sided unpaired $t$ test (A-F), 2 -sided paired $t$ test (I). ${ }^{*} P<0.01,{ }^{* *} P<$ 0.05 (D). Scale bar: $50 \mu \mathrm{m}(\mathbf{H})$.

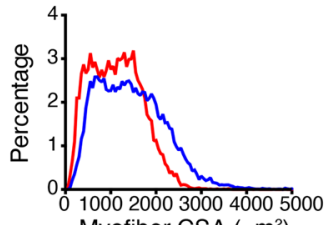

Myofiber CSA $\left(\mu \mathrm{m}^{2}\right)$

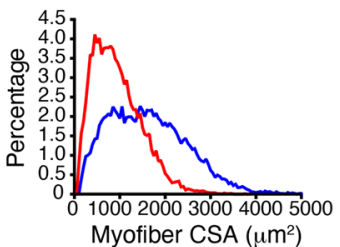

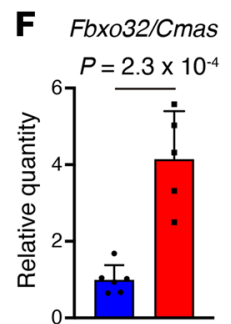

G $\quad \underset{\text { mascle }}{\mathrm{Tg}}$
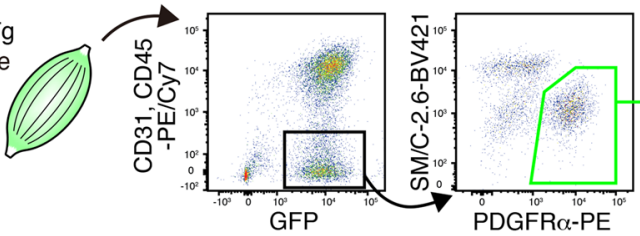

$P_{\alpha}-C E / R 26-D T A$

TA muscle

GFP P $\alpha$ GFP P $\alpha$ GFP P $\alpha$

or PBS or PBS or DiD

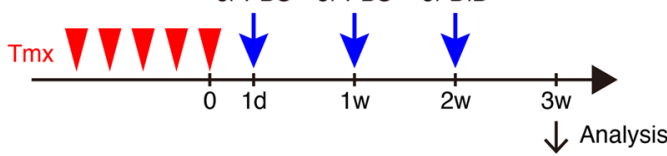

H
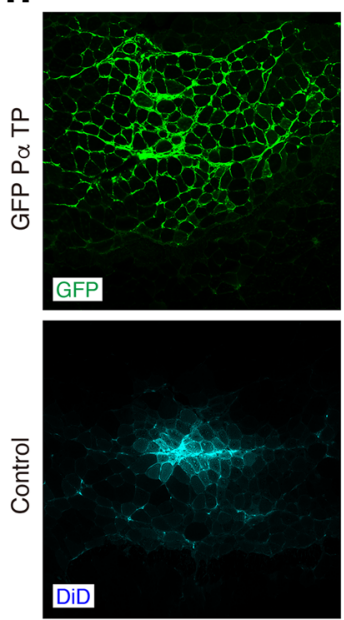

PDGFR $\alpha$
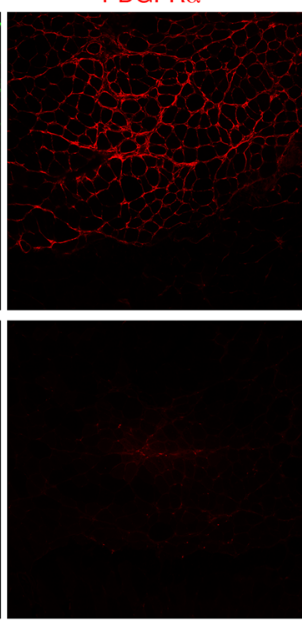

Merge with Laminin $\alpha 2$

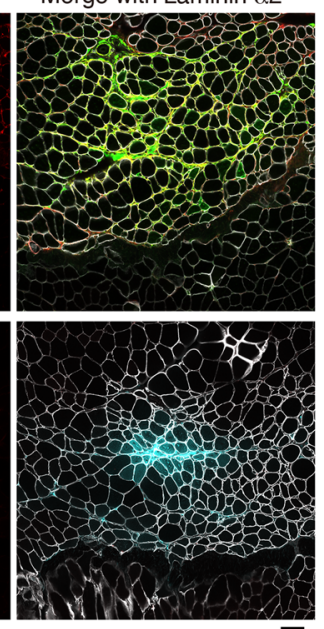

I

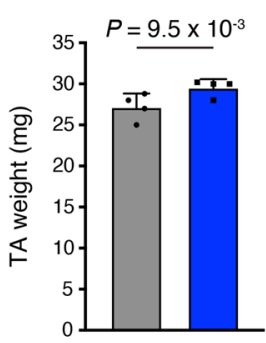


$\mathrm{FR}^{+}$cell ratio among $\mathrm{EYFP}^{+}$cells of $99.7 \% \pm 0.98 \%, n=3$ mice) (Supplemental Figure 1, A and B; supplemental material available online with this article; https://doi.org/10.1172/JCI139617DS1). PDGFR $\alpha^{+}$cells were localized in the interstitial space (Supplemental Figure 1C) and were observed more frequently in the perivascular regions (Supplemental Figure 1, D-F), as previously described (5). Next, $P \alpha-C E$ mice were crossed with R26-DTA mice to deplete mesenchymal progenitors (Figure 1A). To exclude the nonspecific effects of Tmx, the same amount of Tmx was injected into both $P \alpha-C E / R 26-D T A$ mice and WT/R26-DTA control littermate mice. Following Tmx administration, the majority of PDGFR $\alpha^{+}$cells disappeared ( $>80 \%$ decline in PDGFR $\alpha$ intensity), with this reduction lasting 6 or more weeks (Figure 1, B and C), indicating that the ablation of PDGFR $\alpha^{+}$cells cannot be recovered during this time period. FACS analysis revealed that the number of PDGFR $\alpha^{+}$cells specifically decreased by $73 \%$ (Figure $1, \mathrm{D}$ and $\mathrm{E}$ ). This decline rate was underestimated because we normalized cell number by muscle weight yet $P \alpha-C E / R 26-D T A$ mouse muscle weight significantly decreased, as described below. Thus, mesenchymal progenitors were specifically and efficiently depleted in $P \alpha-C E / R 26-$ DTA mice. The depletion of mesenchymal progenitors resulted in reduced body weight, which cannot be attributed to decreased food intake (Figure 2, A and B), along with a significant reduction in muscle strength and weight (Figure 2, C and D). Although the number of myofibers remained unchanged, $P \alpha-C E / R 26-$ $D T A$ mouse myofibers demonstrated reduced cross-sectional area (CSA) at least in histological assessment (Figure 2E). These phenotypes lasted for 6 or more weeks, as depleted mesenchymal progenitors did not replenish. In association with the loss of muscle mass, we observed the upregulation of muscle-specific E3 ubiquitin ligase Fbxo32, which is also known as MAFbx/atrogin-1, in $P \alpha-C E / R 26-D T A$ muscle (Figure $2 \mathrm{~F}$ ). To examine the potential toxicity of killed mesenchymal progenitors toward bystander myofibers, myofiber damage was visualized using Evans blue dye (EBD) or IgG staining (20). No EBD- or IgG-positive myofibers were observed in the $P \alpha-C E / R 26-D T A$ muscle, and we observed only weak interstitial IgG staining (Supplemental Figure 2). In contrast, the intracellular accumulation of EBD and IgG in damaged myofibers was prominent along with the substantial interstitial accumulation of IgG in the dystrophic D2-mdx muscle, which was used as a positive control (Supplemental Figure 2). Thus, no apparent myofiber damage or inflammation in $P \alpha-C E / R 26-D T A$ muscle was observed. As $\mathrm{PDGFR}^{+}$cells also reside in tissues other than skeletal muscle, systemic deletion of PDGFR $\alpha^{+}$cells could influence the $P \alpha-C E / R 26-D T A$ muscle. To address this issue, we reconstituted mesenchymal progenitors specifically in the muscles of the mice previously depleted of mesenchymal progenitors. We transplanted freshly isolated mesenchymal progenitors from GFPTg mice into the tibialis anterior (TA) muscle of the $P \alpha-C E / R 26-$ $D T A$ mice (Figure 2G), resulting in successful engraftment (Figure $2 \mathrm{H}$ ). Transplanted cells were exclusively distributed in the interstitial space while maintaining PDGFR $\alpha$ expression, and they did not contribute to myofibers directly (Figure $2 \mathrm{H}$ ). Notably, the reconstitution of mesenchymal progenitors led to the recovery of muscle weight and fiber CSA specifically in the transplanted muscle (Figure 2I). Wosczyna et al. also demonstrated the importance of muscle-resident mesenchymal progenitors by depleting these cells locally in a specific muscle (14). Our results, together with the results demonstrated by the study conducted by Wosczyna et al., strongly suggest that muscle-resident mesenchymal progenitors are indispensable for steady-state muscle maintenance.

Mesenchymal progenitors play a role in maintaining muscle fiber type and neuromuscular junctions. To gain further insight into the effect of mesenchymal progenitor depletion, we examined the number of satellite cells. The satellite cell number remained unchanged 2 days after the depletion of mesenchymal progenitors (Figure 1E). We did not observe any difference in the number of sublaminar satellite cells even 6 weeks after mesenchymal progenitor depletion (Figure 3A). However, a previous study demonstrated that the number of satellite cells decreased 9 months after the depletion of mesenchymal progenitors (14). Hence, the mesenchymal progenitors appear to be required for the long-term maintenance of satellite cells. Muscle wasting is frequently accompanied by fiber type shift; moreover, fast-to-slow shifts also occur during aging (21). Therefore, we examined the fiber type of mesenchymal progenitor-depleted mice. Analysis of TA muscle fiber type, typically consisting of predominantly fast fibers, demonstrated an increased ratio of slow fibers in $P \alpha-C E / R 26-D T A$ mice (Figure 3, B and $C$ ), although the change was small.

As proper muscle-nerve interaction constitutes a critical factor in the maintenance of muscular health, we examined the relationship between mesenchymal progenitors and motor nerves. Whole-mount immunostaining revealed that mesenchymal progenitors surround the motor nerve axon and also cover the neuromuscular junction (NMJ) in wild-type (WT) mice (Figure 4A), with the majority of postsynaptic endplates being completely occupied by presynaptic nerve terminals (Figure 4B). However, $P \alpha-C E / R 26-D T A$ mice exhibited an increased ratio of partially or completely denervated NMJs, and this ratio tended to increase at later time points (Supplemental Figure 3 and Figure 4B). We also observed that the mesenchymal progenitors were located adjacent to Schwann cells, whereas $P \alpha-C E / R 26-D T A$ mice exhibited disorganized Schwann cells (Figure $4 \mathrm{C}$ ). Consistent with these morphological changes, negative regulators of Schwann cell differentiation, such as Jun and SRY-box 2 (Sox2), were upregulated in $P \alpha-C E / R 26-D T A$ mice, and the expression of inhibitor of DNA binding 2 ( $I d 2)$, another negative regulator, was also likely to increase, although not to a statistically significant extent (Figure 4D). Conversely, mature Schwann cell markers (myelin basic protein $[M b p]$ and gap junction protein $\beta-1[G j b 1]$ ) were downregulated (Figure 4D). Interestingly, similar deterioration of NMJ and Schwann cells was also observed in sarcopenic geriatric mice (22).

Bmp3b is a mesenchymal progenitor-specific and sarcopenia-related gene. As $P \alpha-C E / R 26-D T A$ mouse phenotypes were markedly similar to the phenotype observed in sarcopenic conditions, we sought to identify factors important for mesenchymal progenitordependent muscle maintenance and involved in aging-related sarcopenia. We first characterized mesenchymal progenitors in aged mice. Mesenchymal progenitors were observed to reside in the muscle interstitial space of both young and aged mice in a similar manner (Figure 5A). However, in the FACS analysis, we observed a clear decrease in the PDGFR $\alpha^{+}$fraction and a minor decrease in PDGFR $\alpha$ fluorescence intensity in aged mice (Figure 5B). Moreover, aged mesenchymal progenitors showed an increase in the 
A
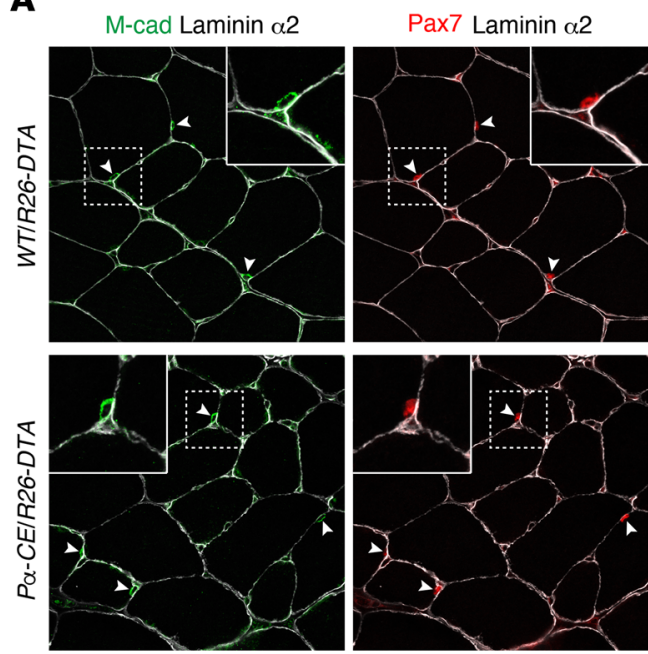

B

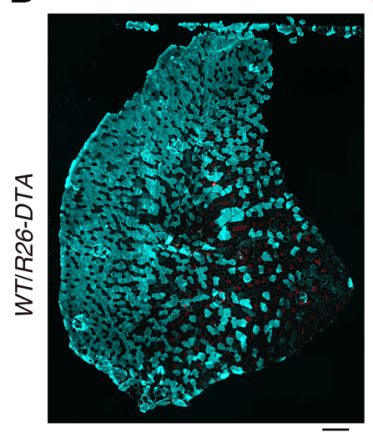

Type I Type IIB

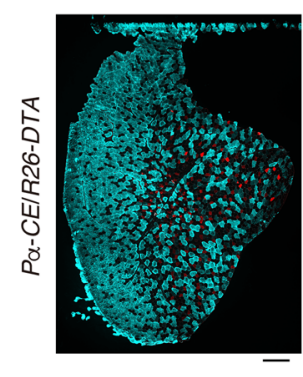

Type IIA
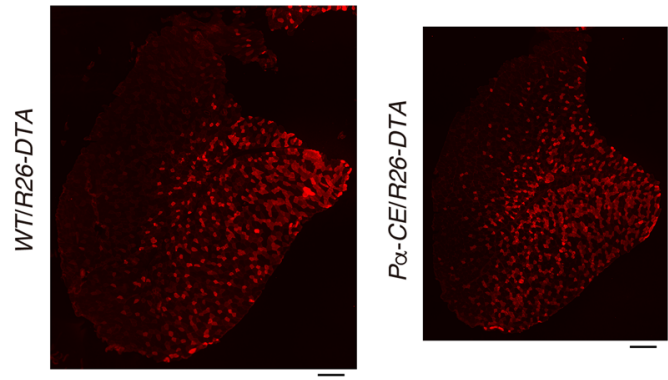

M-cad Pax7 Laminin $\alpha 2$ DAP
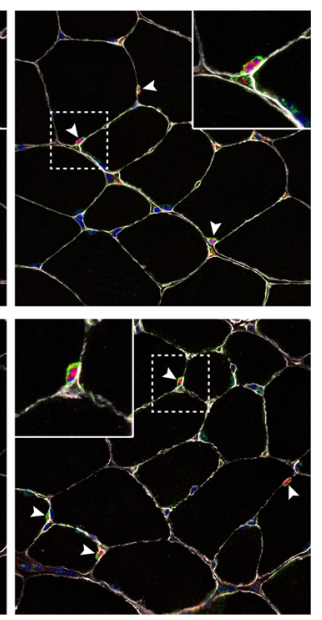

- WT/R26-DTA - $P_{\alpha}-C E / R 26-D T A$

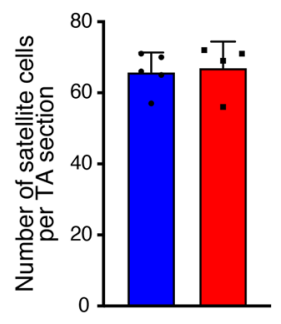

Figure 3. Effect of mesenchymal progenitor depletion on satellite cells and myofiber type. (A) Immunostaining of TA muscle for $M$-cadherin ( $M$-cad), Pax7, and laminin $\alpha 2$. The number of satellite cells was quantified. Arrowheads: satellite cells. Inset: the magnified image of the boxed region. $n=5$ for $W T / R 26$ DTA and $n=4$ for $P \alpha-C E / R 26-D T A$. (B) Fluorescence images of type I, type IIB, and type IIA myosin heavy chain in TA muscle. (C) Percentage of each fiber type. $n=4$ for WT/R26-DTA and $n=5$ for $P \alpha-C E / R 26-D T A$. Data represent the mean \pm SD; 2-sided unpaired $t$ test. Scale bars: $20 \mu \mathrm{m}$ (A) and $250 \mu \mathrm{m}$ (B).

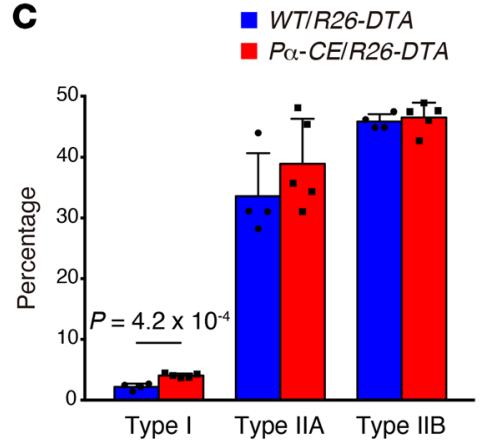

WT/R26-DTA side scatter (SSC) profile (Figure 5B). Accordingly, mesenchymal progenitor numbers decreased with aging (Figure $5 \mathrm{C}$ ). These results indicate the presence of certain differences in the quality of cells in addition to the cell number between young and aged mesenchymal progenitors.

Subsequently, we searched for genes with decreased expression in $P \alpha-C E / R 26-D T A$ muscle compared with those in the control (Figure 5D). As the depletion of mesenchymal progenitors leads to muscle weakness and atrophy, genes that are functionally important for muscle maintenance and are expressed by mesenchymal progenitors must be selected. However, as genes expressed in other cell types such as satellite, endothelial, and hematopoietic cells might also be included since whole muscle tissue was used as a sample, we performed further analysis using FACS-purified cells to identify mesenchymal progenitor-specific genes that were also downregulated in aged mice (Figure 5D). Among genes fulfilling both criteria, we focused on
$B m p 3 b$ since it is the only factor that exhibits cytokine or growth factor activity based on Gene Ontology and hence assumed to be a candidate molecule responsible for mesenchymal progenitor-dependent muscle maintenance (Figure 5D and Supplemental Table 1). BMP3B, a member of the TGF- $\beta$ superfamily, is also known as growth differentiation factor 10 (GDF10). We first confirmed that $B m p 3 b$ is specifically expressed by mesenchymal progenitors and significantly downregulated in the $P \alpha-C E / R 26-D T A$ muscle or aged mesenchymal progenitors (Figure 5, E and F). Bmp3b expression in muscle tissue was also significantly decreased in aged muscle in both mice (Figure 5G) and humans (Figure 5H). Furthermore, freshly isolated mesenchymal progenitors demonstrated a high Bmp3b expression. However, this expression was decreased after in vitro culture and further decreased upon adipogenic differentiation (Figure 5I). PDGFR $\alpha^{+}$mesenchymal progenitors were also identified in other tissues including the heart $(23,24)$ and bone marrow $(25)$, 
A
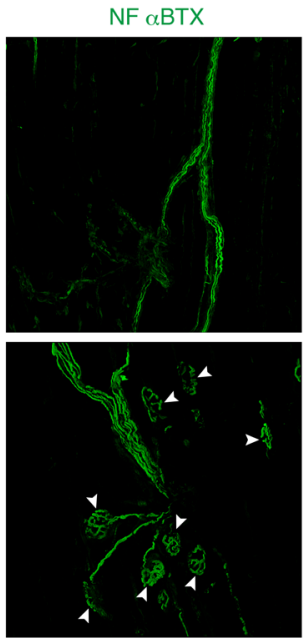

PDGFR $\alpha$
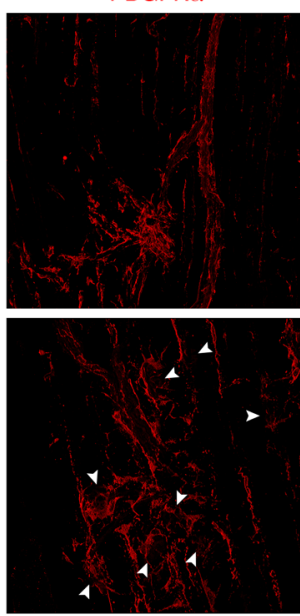

NF aBTX PDGFR $\alpha$ DAPI
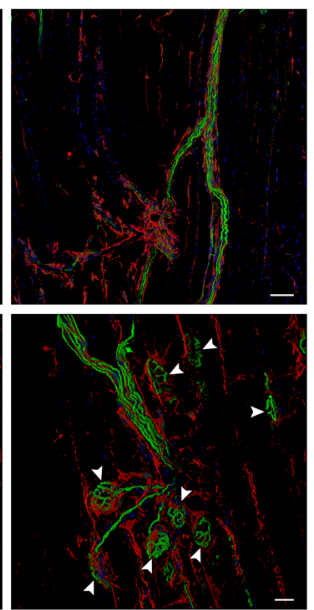

Figure 4. Mesenchymal progenitor depletion results in defects in neural components. (A) Whole-mount immunofluorescence staining of WT EDL muscle for neurofilament $(\mathrm{NF})$, acetylcholine receptor ( $\alpha \mathrm{BTX})$, and PDGFR $\alpha$. Arrowheads: NMJs. (B) Fluorescence images of acetylcholine receptor $(\alpha \mathrm{BTX})$ and synaptophysin. Insets show magnified images of boxed regions. Ratios of completely denervated (Comp. den), partially denervated (Part. den), and innervated (In) NMJ were calculated at 17 days after Tmx treatment. $n=6$ for WT/R26-DTA, $n=8$ for $P \alpha-C E / R 26$ DTA. (C) Whole-mount immunofluorescence staining for PDGFR $\alpha$, S100, and $\alpha$ BTX. (D) Expression of Schwann cellrelated genes in the mid-belly of the TA muscle. $n=6$ for WT/R26-DTA and $n=5$ for $P \alpha-C E / R 26-D T A .{ }^{*} P<0.01,{ }^{*} P$ $<0.05$. Data represent the mean \pm SD; 2 -sided unpaired $t$ test. Scale bars: $20 \mu \mathrm{m}$ (A: lower panels), $50 \mu \mathrm{m}$ (A: upper panels, and C), and $75 \mu \mathrm{m}$ (B).
B

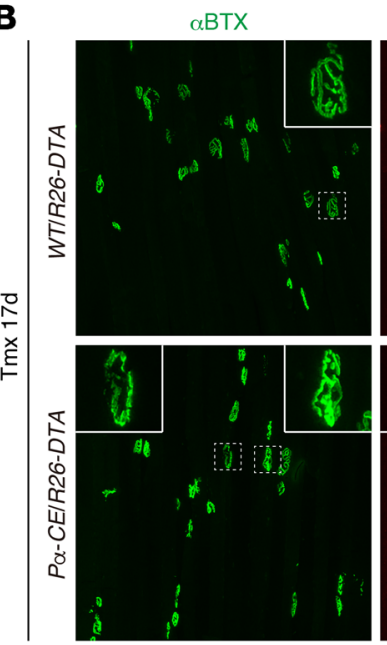

Synaptophysin

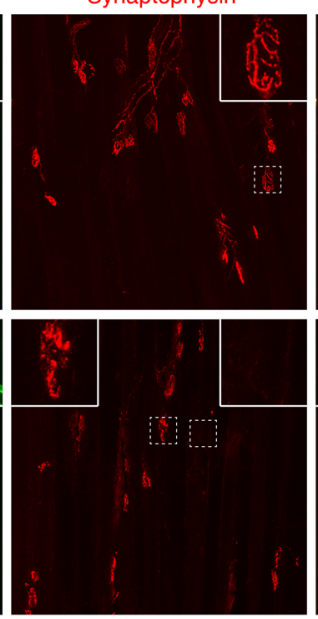

Merge

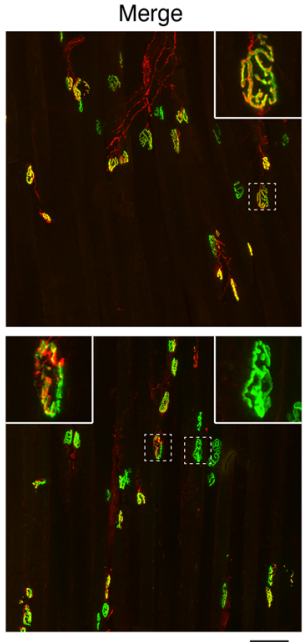

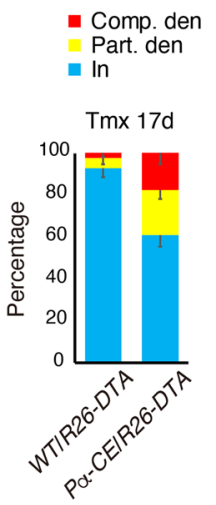
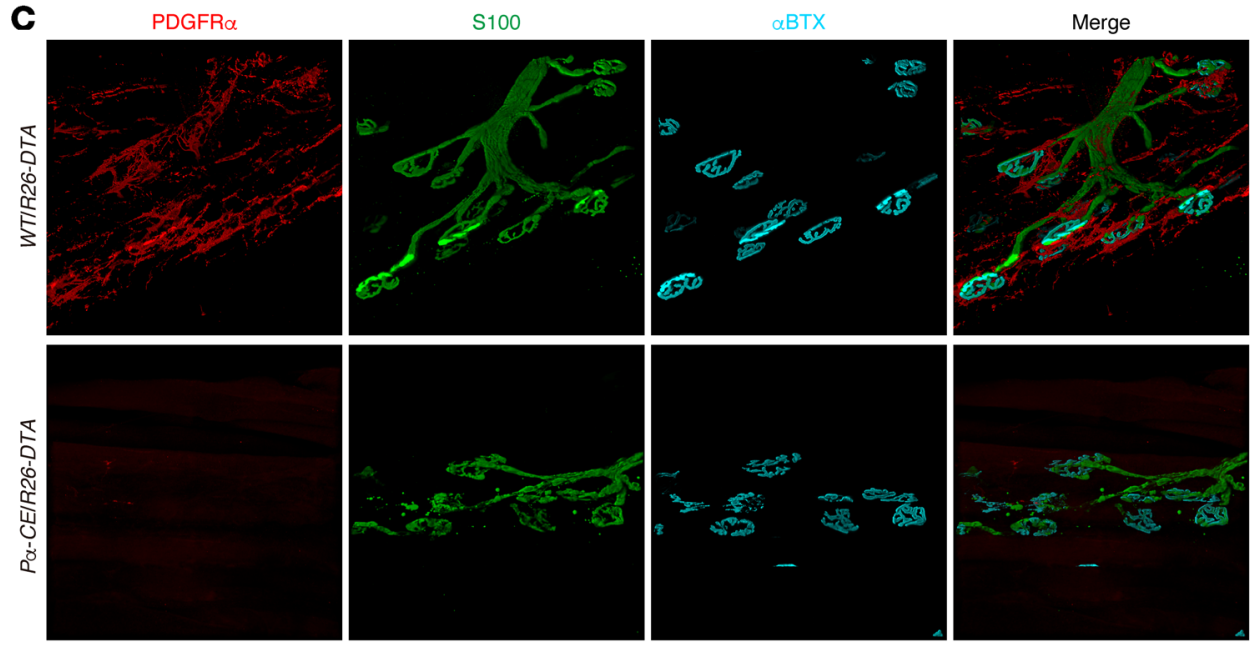

D WTIR26-DTA $\square$ - PQ-CE/R26-DTA

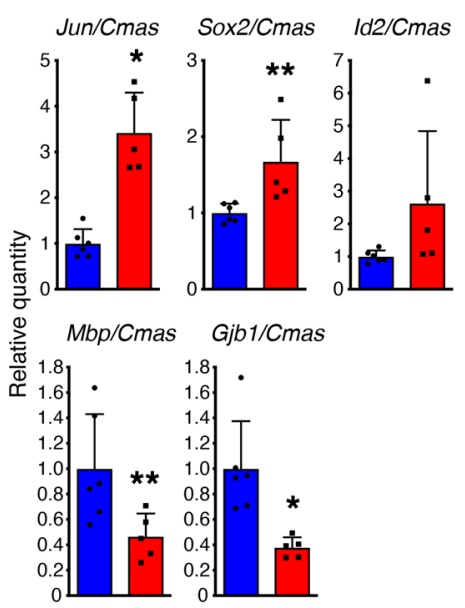

although these expressed $B m p 3 b$ at a lower level than that by muscle-resident mesenchymal progenitors (Figure 5J).

Mesenchymally expressed Bmp3b is functionally important for the maintenance of muscle health. To elucidate the function of BMP3B, we examined Bmp3b-KO mice (26). Careful examination of the $\mathrm{KO}$ mouse phenotype demonstrated a minor but statistically signifi- cant reduction in body weight (Figure 6A) along with decreased muscle weight, which can be attributed to reduced myofiber CSA rather than the reduced number of myofibers (Figure 6, B and C). A decrease in the grip strength was also observed in $\mathrm{Bmp} 3 \mathrm{~b}-\mathrm{KO}$ mice, despite normalization by body weight (Figure 6D), suggesting that functional decline is not simply due to decreased body 
A
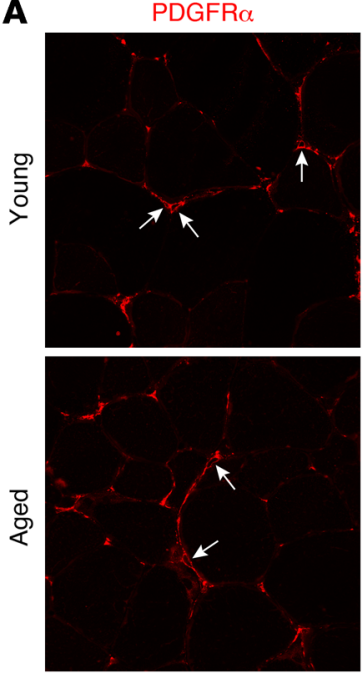

B

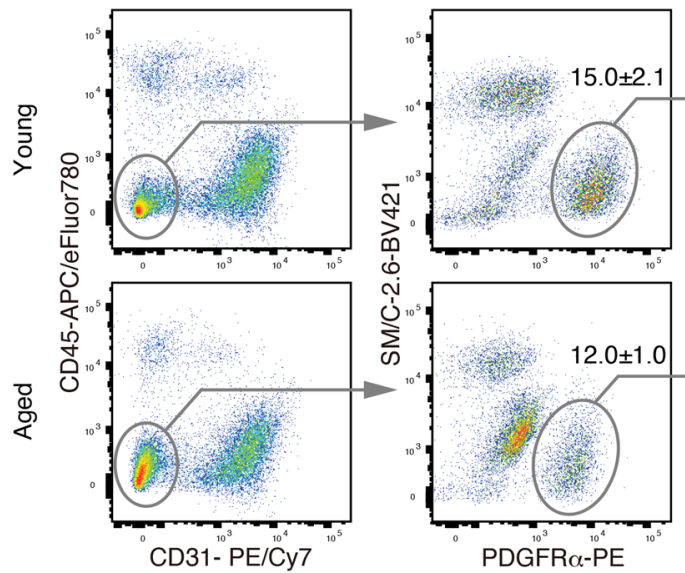

D

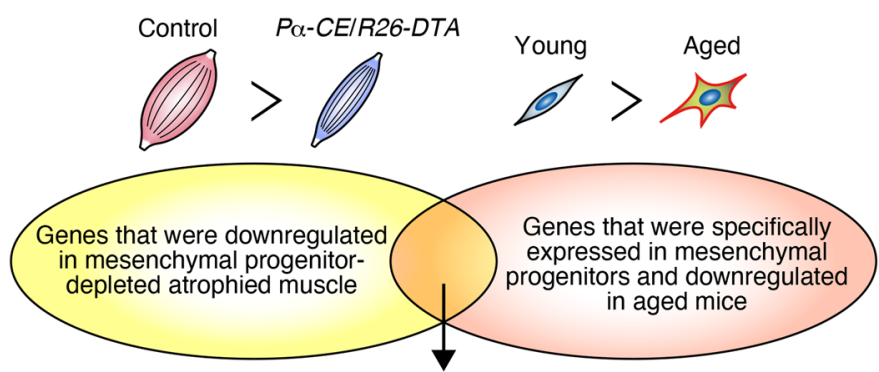

Bmp3b

G

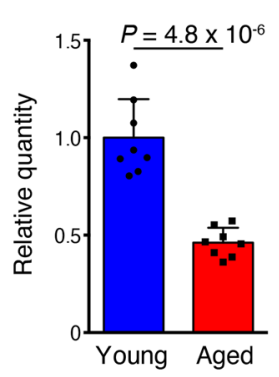

Laminin $\alpha 2$
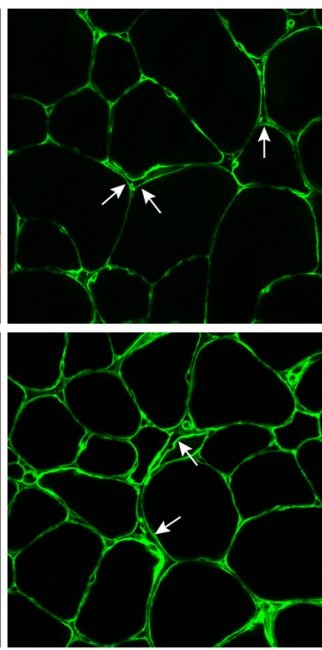

CD31-CD45
H BMP3B/NDUFA13 I

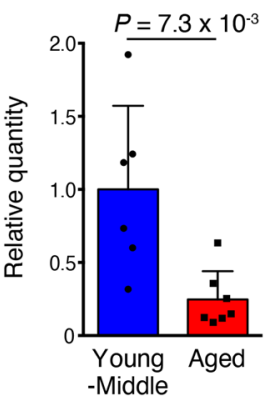

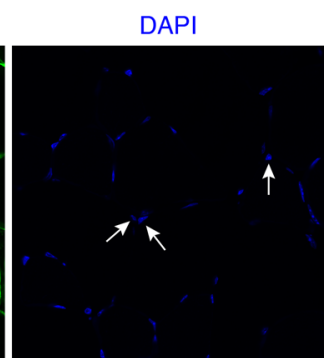

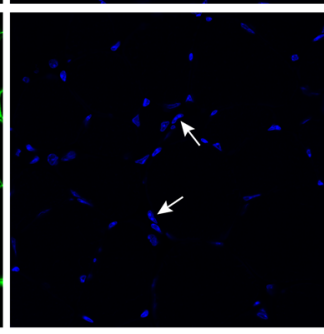

PDGFR $\alpha^{+}$

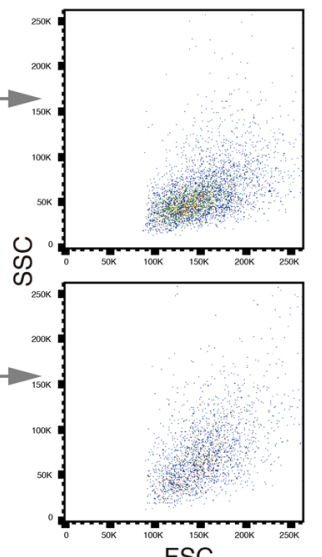

E

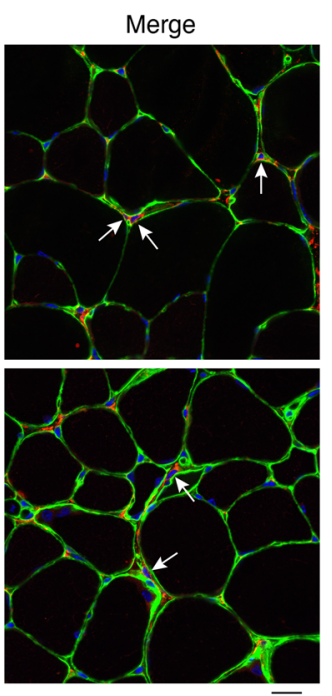

PDGFR $\alpha^{+}$

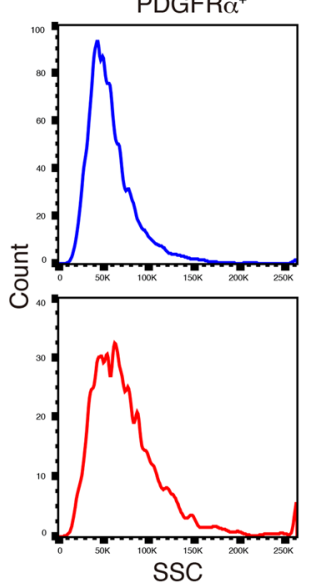

C

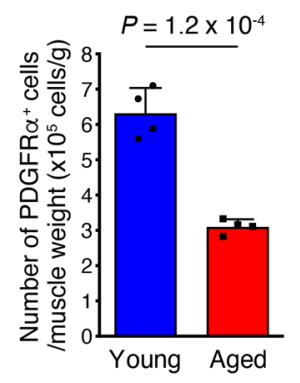

Bmp3b/Hsbp 1

$\mathbf{F}$

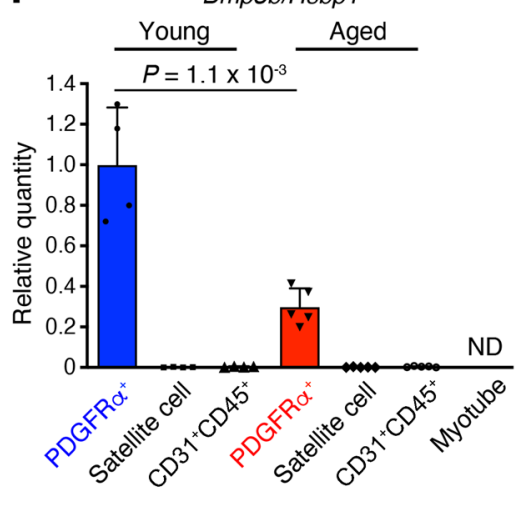

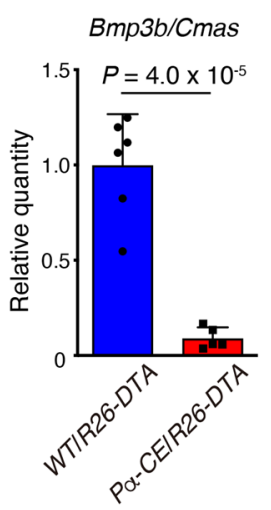

Bmp3b/Hsbp1

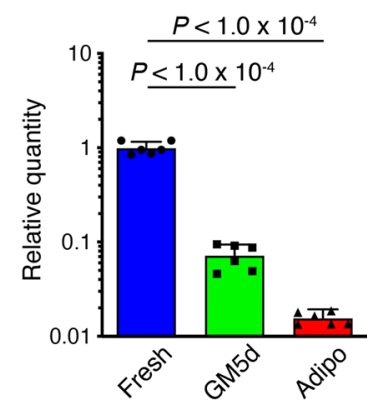

Bmp3b/Hsbp 1

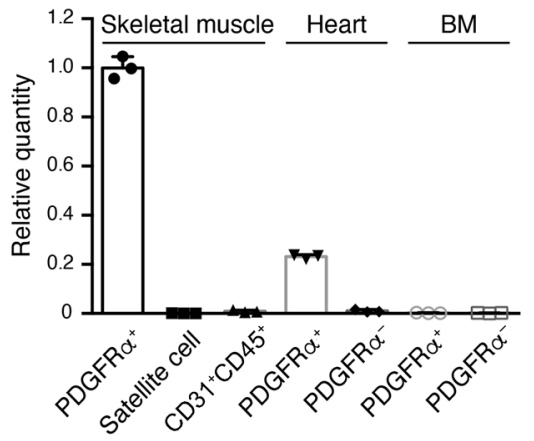


Figure 5. Identification of $B m p 3 b$ as a mesenchymal progenitor-specific and sarcopenia-related gene. (A) PDCFR $\alpha$ and laminin $\alpha 2$ fluorescence images of young and aged muscles. Arrows indicate PDCFR $\alpha$ cells located in the interstitial space. (B) FACS analysis of young and aged muscle. The percentage of PDGFR $\alpha^{+}$cells in the total mononucleated cells is shown. $n$ $=4$. (C) Cell number per muscle weight. $n=4$. (D) Scheme for the identification of genes responsible for mesenchymal progenitor-dependent muscle maintenance. (E) Expression of $B m p 3 b$ in the TA muscle. $n=6$ for $W T / R 26-D T A$ and $n=5$ for $P \alpha-C E / R 26-D T A$. (F) Expression of Bmp3b in FACS-isolated cell populations. As myofibers were difficult to sort, satellite cell-derived myotubes were used. ND, not detected. $n=4$ for young, $n$ $=5$ for aged. (G) Expression of $B m p 3 b$ in the TA muscles of young and aged mice. $n=8$ mice. $(\mathbf{H})$ Comparison of $B m p 3 b$ expression in human muscle between young-middle-aged and aged groups. $n=6$ for young-middle, $n=$ 7 for aged. (I) Bmp3b expression in mesenchymal progenitors was analyzed immediately after sorting (Fresh), after 5 days of culture (GM5d), and after adipogenic differentiation (Adipo). $n=6$ independent samples. (J) $B m p 3 b$ expression in FACS-purified cells from the skeletal muscle, heart, and bone marrow. $n=3$ independent samples. Data represent the mean $\pm S D$; 2-sided unpaired $t$ test (C and $\mathbf{E}-\mathbf{H}$ ), ANOVA followed by Dunnett's test (I). Scale bar: $20 \mu \mathrm{m}$ (A).

weight. In addition, as the weight of other organs such as the liver and adipose tissue was comparable to that of WT (Supplemental Figure 4), the observed muscle phenotype could not be simply attributed to growth deficit. Bmp3b-KO mice showed a tendency of a minor increase in slow fibers and an increase in type IIA fibers (Figure 6, E and F). We also observed NMJ degeneration (Figure $6 \mathrm{G}$ ) and disorganized Schwann cells (Figure $6 \mathrm{H}$ ) in Bmp3b-KO mice, although these phenotypes were less severe than those in the $P \alpha-C E / R 26-D T A$ mice.

To gain insight into the function of BMP3B, we used a culture system of FACS-purified cells. Purified WT satellite cells were cultured alone or cocultured with PDGFR $\alpha^{+}$cells purified from WT, $B m p 3 b-\mathrm{KO}$, or $P \alpha-C E / C A G-C A T-m B m p 3 b-\mathrm{Tg}$ (hereafter referred to as $P \alpha / B m p 3 b-\mathrm{Tg}$ ) mice (27), in which $B m p 3 b$ overexpression was specifically induced in PDGFR $\alpha^{+}$cells (Supplemental Figure $5 \mathrm{~A})$. Coculture with WT PDGFR $\alpha^{+}$cells resulted in the formation of larger myotubes than those in the satellite cell single culture (Figure 7A). Although not statistically significant, the myotube area demonstrated a tendency to increase in coculture with WT PDGFR $\alpha^{+}$cells compared with that in coculture with $B m p 3 b-\mathrm{KO}$ PDGFR $\alpha^{+}$cells (Figure 7A). Similar results were observed when conditioned media (CM) harvested from PDGFR $\alpha^{+}$cells of each genotype was added to mature human myotubes (Supplemental Figure $5 \mathrm{~B}$ and Figure $7 \mathrm{~B}$ ). Since $\mathrm{Bmp} 3 \mathrm{~b}$ is downregulated after in vitro culture (Figure 5I), we used $P \alpha / B m p 3 b$-Tg PDGFR $\alpha^{+}$cells to make the effect of BMP3B clearer. The degree of hypertrophy in myotubes became more pronounced in coculture with $P \alpha / B m p 3 b$ Tg PDGFR $\alpha^{+}$cells (Figure 7A), and CM harvested from $P \alpha / B$ $m p 3 b$-Tg PDGFR $\alpha^{+}$cells induced myotube hypertrophy more efficiently compared with that harvested from WT or Bmp3b-KO PDGFR $\alpha^{+}$cells (Figure 7B), with accompanying weak Smad2 and more obvious Smad1/-5/-8 activation (Supplemental Figure 5C). To elucidate whether BMP3B influences muscle differentiation, we treated mouse satellite cells with recombinant BMP3B during the first day of differentiation to examine its effect on early differentiation response. No difference was observed in the myogenin $^{+}$cell ratio and fusion index between BMP3B-treated cells and control cells (Figure 7C), indicating that BMP3B does not affect muscle differentiation. This observation was further supported by the significantly decreased expression of $B m p 3 b$ in mesenchymal progenitors derived from muscle injected with cardiotoxin (CTX), where active myogenesis was occurring, compared with the cells derived from intact muscle (Figure 7D). The treatment of fully differentiated human myotubes with BMP3B resulted in an induction of myotube hypertrophy without affecting the fusion index (Figure 7E) along with a moderate activation of Smad1/-5/-8 and the Akt pathway, which is the signaling pathway involved in the maintenance of muscle mass (28-31), and no effect on Smad2 (Figure $7 F)$. Conversely, Smad1/-5/-8 and Akt signaling were attenuated in atrophied $B m p 3 b-\mathrm{KO}$ muscle (Figure $7 \mathrm{G}$ ). These results suggest that BMP3B preserves muscle mass by directly acting on differentiated myofibers.

Next, we investigated the effect of BMP3B on Schwann cells, as mesenchymal progenitor depletion and $B m p 3 b$ deletion resulted in Schwann cell abnormalities. BMP3B suppressed Jun expression in rat primary Schwann cells (Figure 8A). Although Mbp expression was not affected, BMP3B induced the upregulation of early growth response 2 (Egr2), an essential regulator of the myelination program (Figure 8A). In vitro myelination assays performed with murine dorsal root ganglia demonstrated the promotion of myelination by BMP3B (Figure 8B). Hence, BMP3B seems to positively regulate the characteristics of Schwann cells by stabilizing the differentiated state.

$B M P 3 B$ improves energy metabolism and increases muscle mass and strength in aged mice. Since the expression of $B m p 3 b$ was significantly decreased in both purified mesenchymal progenitors and muscle tissue during aging, we administered BMP3B to aged mice. After 2 weeks of administration, we performed several physiological and metabolic assessments. BMP3B-treated mice exhibited increasing tendencies of oxygen consumption and carbon dioxide production, and accordingly demonstrated an increased energy expenditure in the light period (Figure 9, $\mathrm{A}$ and $\mathrm{B})$. However, the locomotive activity did not differ significantly (Figure 9C). The administration of BMP3B did not affect body weight (Figure 9D) but improved the grip strength (Figure 9E) and augmented some of the muscle weight-consequent myofiber hypertrophy (Figure 9, F and G), indicating that BMP3B can reverse or inhibit certain phenotypes of sarcopenia. These results suggest that the reduction of mesenchymally expressed BMP3B in the aged muscle represents a contributing factor in the development of sarcopenia.

\section{Discussion}

In this study, we describe the essential functions of interstitial mesenchymal progenitors for the maintenance of muscle integrity under steady-state conditions. Although satellite cells contribute to myofibers with varying extent and timing, satellite cell depletion experiments showed that they are not required globally for the maintenance of myofibers during aging $(17,18)$. Conversely, mesenchymal progenitor-depleted mice exhibited premature aging phenotypes that were markedly similar to sarcopenia. A recently published paper also demonstrated the importance of mesenchymal progenitors in homeostatic muscle maintenance by depleting these cells, but the mechanism by which mesenchymal progeni- 
A

B
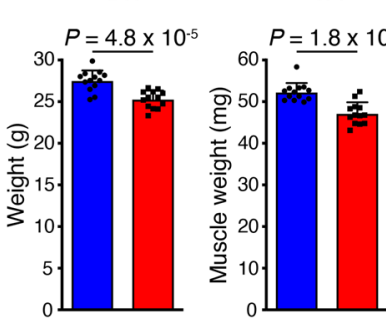

Type I Type 118
C

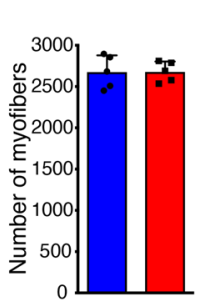

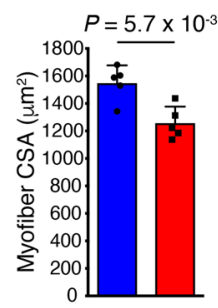

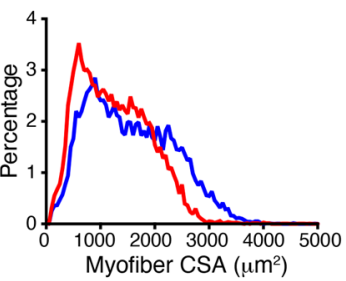

D Grip strength $\quad$ WO

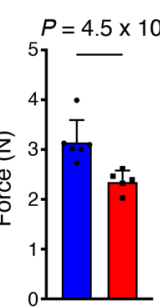
$P=1.4 \times 10^{-2}$
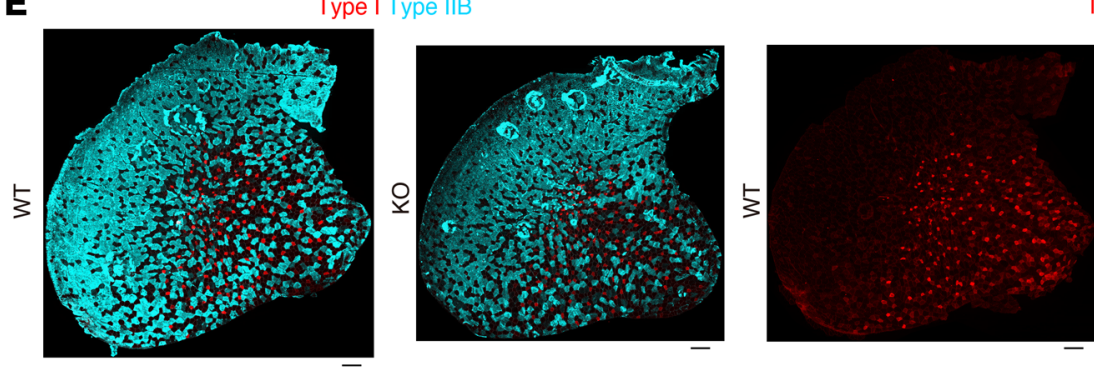

Type IIA

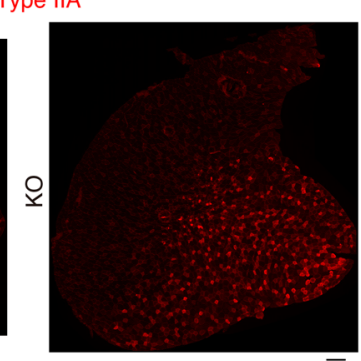

$\mathbf{F}$

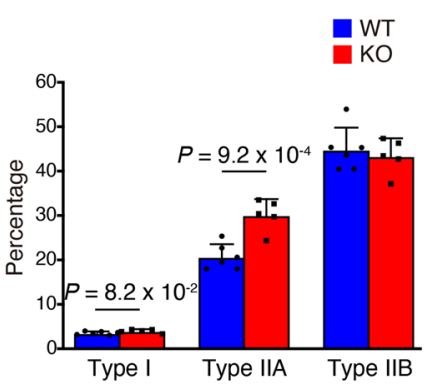

G

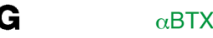

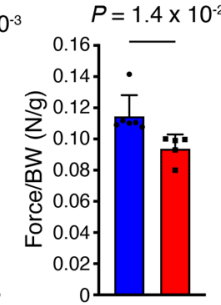


tors maintain muscle remains to be elucidated (14). In the present study, we comprehensively explored factors critical for mesenchymal progenitor-dependent muscle maintenance. Consequently, we concluded that the beneficial effects of mesenchymal progenitors can be explained, at least in part, by the roles of the mesenchymal progenitor-specific gene $B m p 3 b$ described in the current study. Here, we demonstrated that BMP3B directly ensures muscle integrity by stimulating signals important for muscle mass maintenance. We also uncovered an unexpected relationship between mesenchymal progenitors and neural components, along with the underlying mechanisms by which BMP3B stabilizes the characteristics of Schwann cells. Since Schwann cells are required for the formation and maintenance of the NMJ (32), mesenchymally derived BMP3B also presumably establishes muscle health indirectly via its action on Schwann cells and the NMJ. Mesenchymal progenitor-depleted mice and $B m p 3 b$-KO mice showed minor changes in muscle fiber type. However, it remains to be elucidated whether the alterations in fiber type can be attributed to the diminished direct action of BMP3B on myofibers or secondary response to the deterioration of neural components. Collectively, our study highlights the critical role of stromal components in sustaining parenchyma, raising the possibility of the broader importance of such mesenchymal-parenchymal interactions in the maintenance of homeostasis in diverse tissues.

Although we revealed that BMP3B is specifically expressed in PDGFR $\alpha^{+}$cells within the skeletal muscle, we cannot exclude the effect on muscle maintenance of BMP3B expressed in the cells residing in other tissues. However, previous studies have demonstrated the local paracrine action of the TGF- $\beta$ superfamily. One of the well-known functions of the TGF- $\beta$ superfamily is the regulation of epithelial-mesenchymal transition (EMT). The activation of EMT is mediated via a series of paracrine signaling molecules such as TGF- $\beta$ families, including BMP3B $(33,34)$. It is also well known that TGF- $\beta$ superfamily members, especially BMPs, act in the restricted region during development. Some BMPs have been reported to influence and induce ventral fates only in the regions in which they are expressed, and the action of BMPs is tightly restricted to the regions within and around the cells that produce them (35). Our local reconstitution experiment and previous local depletion experiment support this paracrine action. We observed the recovery of $P \alpha-C E / R 26-D T A$ muscle specifically in mesenchymal progenitor-transplanted muscle but not in the contralateral muscle, while muscle atrophy induced by the local depletion of mesenchymal progenitors cannot be compensated by remaining cells nearby (14). Hence, it seems likely that mesenchymal progenitors and their secretory products act locally where they reside or are expressed. In addition, we observed that muscle-resident PDGFR $\alpha^{+}$cells express significantly higher levels of $\mathrm{BMP} 3 \mathrm{~B}$ than those by PDGFR $\alpha^{+}$cells residing in the heart and bone marrow. Recent single-cell analysis of PDGFR $\alpha^{+}$cells from 4 different organs also revealed preferential expression of BMP3B in PDGFR $\alpha^{+}$cells of the skeletal muscle. (36). Given the paracrine action of the TGF- $\beta$ superfamily and preferential expression of BMP3B in muscle-resident PDGFR $\alpha^{+}$cells, $\mathrm{BMP} 3 \mathrm{~B}$ produced in the muscle-resident PDGFR $\alpha^{+}$cells may be responsible for muscle maintenance.
Previous studies have also shown the beneficial function of BMP3B by demonstrating its antiadipogenic action, metabolically favorable influence on obesity (27), and a neuroprotective role following stroke (37). Recent single-cell RNA sequencing (RNA-seq) analysis of skeletal muscle also confirmed the antiadipogenic role of BMP3B (38). Notably, BMP3B expression was decreased during aging in both mice and humans, whereas its administration to aged mice reversed certain phenotypes of sarcopenia, including the improvement of muscle function and mass. BMP3B-treated aged mice showed increased energy expenditure during the light period without the gain of locomotive activity. This may be attributed to an increase in basal metabolism owing to increased muscle mass and the metabolically favorable property of BMP3B (27), although increase in energy expenditure does not necessarily link to health improvement. All these results lead to what we believe is a new concept, which states that age-related changes in mesenchymal progenitors, including reduced BMP3B levels, can be a causal factor of sarcopenia. This concept is further supported by the significantly decreased BMP3B levels in response to adipogenic differentiation and the strong link between the degree of fat infiltration and decline in motor function in elderly individuals (39).

Although we revealed the beneficial function of mesenchymal progenitors in steady-state muscle maintenance, these cells also exert a positive effect on muscle regeneration. Mesenchymal progenitors stimulate the expansion of muscle stem cells and hematopoietic cells and, hence, are required for successful muscle regeneration $(12,14)$. Although the molecular basis for the regeneration-promoting function of these cells is not yet completely understood, WNT1-inducible signaling pathway protein 1 (WISP1) is a mesenchymal progenitor-derived factor that regulates satellite cell-dependent myogenesis and promotes muscle regeneration (40). Recent single-cell RNA-Seq and ATAC-Seq analyses revealed that mesenchymal progenitors express many kinds of trophic factors and extracellular matrix to orchestrate muscle regeneration (41). In addition to their role in muscle regeneration, mesenchymal progenitors also exhibit a pathological role as contributors to fatty and fibrous degeneration. As mesenchymal progenitors have dual contradictory functions $(42,43)$, i.e., promotion of regeneration and the development of pathogenesis, elucidating mechanisms that contribute to the decision of the fate of mesenchymal progenitors would be of considerable interest.

In this study, we investigated the role of PDGFR $\alpha^{+}$mesenchymal progenitors in the skeletal muscle, but similar PDGFR $\alpha^{+}$ progenitors also reside in the heart $(23,24)$ and bone marrow (25). Our results revealed that muscle-resident mesenchymal progenitors demonstrated the highest expression of BMP3B among these sources. Similarly, recent single-cell analysis of PDGFR $\alpha^{+}$cells and mural cells derived from 4 different murine organs identified $B m p 3 b$ as one of the skeletal muscle PDGFR $\alpha^{+}$ cell-specific genes (36). Thus, there is heterogeneity among mesenchymal cell populations, and interorgan heterogeneity suggests a tissue-specific role of them. This hypothesis is further supported by the restricted expression of the chemokine CXCL12, which functions as a niche factor for hematopoietic stem cells. CXCL12 is highly expressed in bone marrow niche cells exhibiting mesenchymal progenitor potential (44) but shows low expression in muscle mesenchymal progenitors (45). 
A

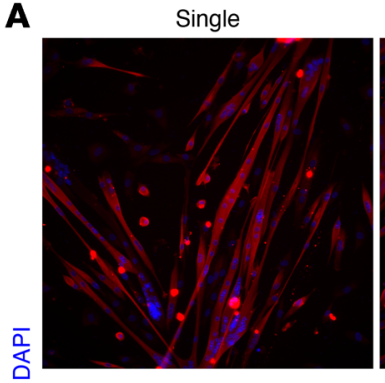

WT P $\alpha$ co-culture
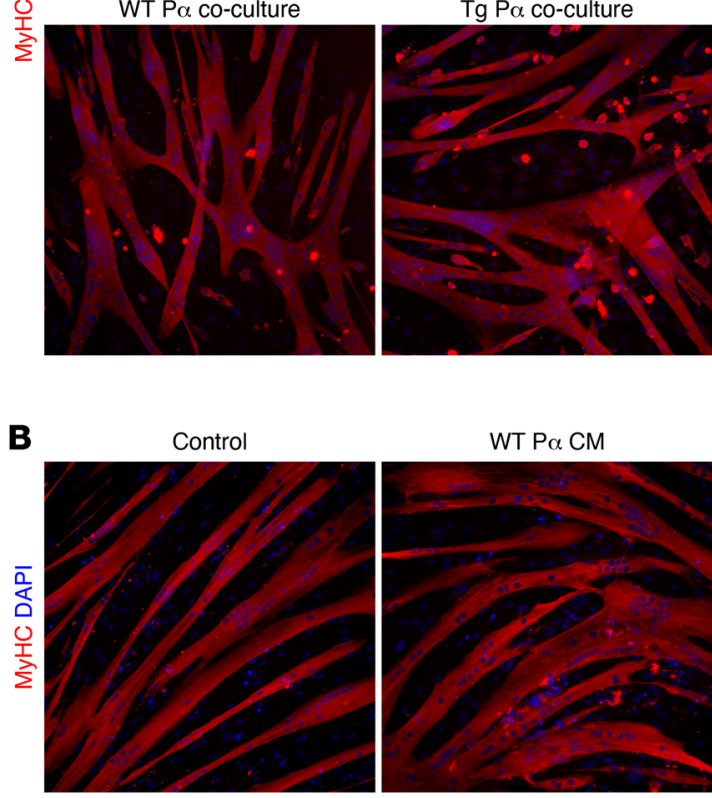

C
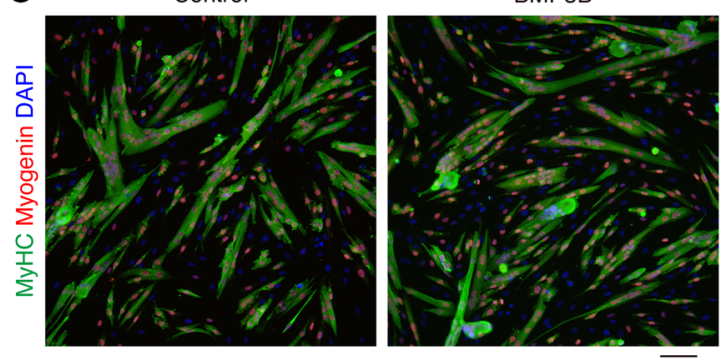
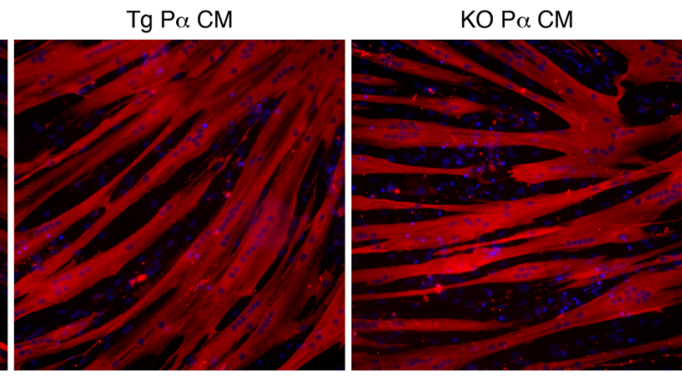

BMP3B
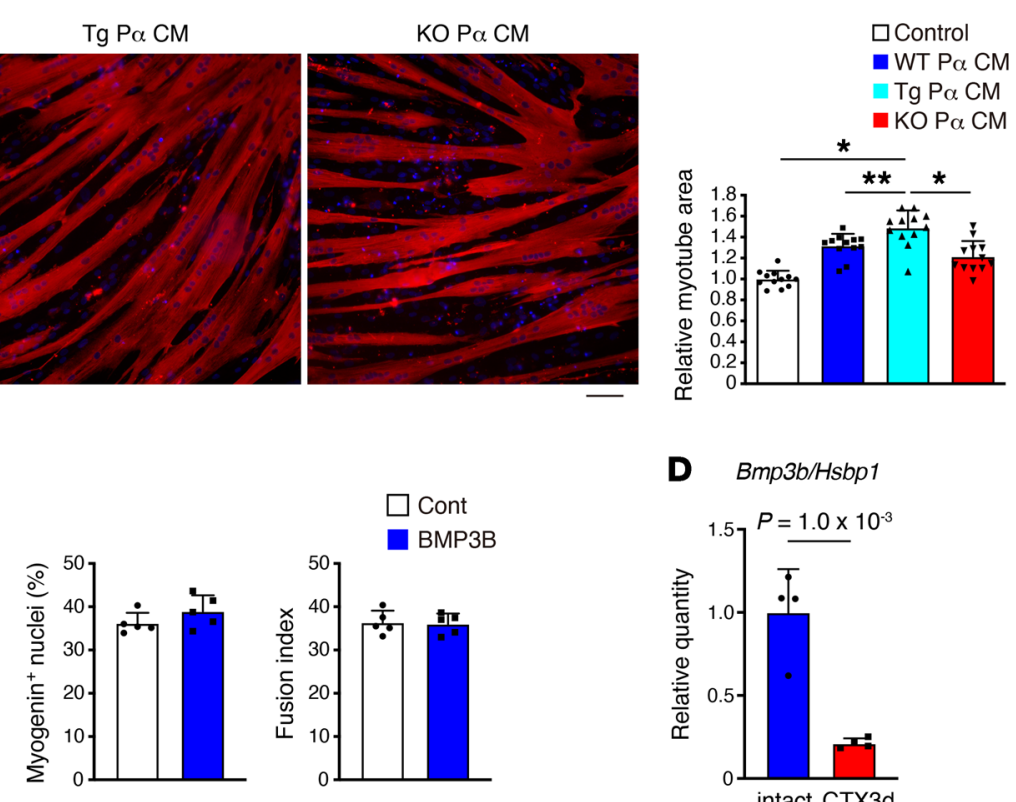

D $\quad B m p 3 b / H s b p 1$

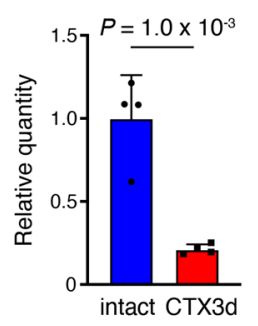

G

p-Smad2

$\mathrm{p}-\mathrm{Smad} 1 / 5 / 8$

p-Akt

$\mathrm{p}-\operatorname{Smad} 1 / 5 / 8 \quad-\mathrm{m}=-\mathrm{m}=0$

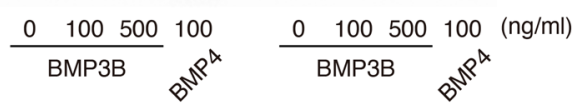

GAPDH

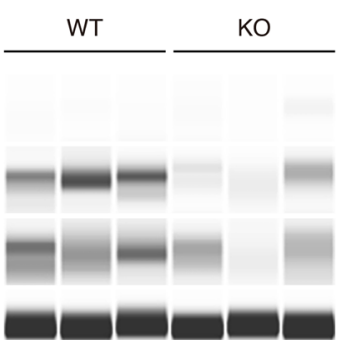

p-Akt

$$
\frac{0 \quad 100500}{\text { BMP3B }} \quad \frac{0100500}{\text { BMP3B }}(\mathrm{ng} / \mathrm{ml})
$$


Figure 7. BMP3B maintains skeletal muscle by stimulating hypertrophic signaling pathway. (A) MyHC staining of satellite cell single culture or coculture. As mesenchymal progenitors promote satellite cell proliferation, single culture using 3 times the number of satellite cells (single $\times 3$ ) was performed to circumvent the proliferation-stimulating effect. $n=12$ randomly selected fields. (B) MyHC staining of human myotubes treated with CM. $n=12$ randomly selected fields. (C) Myogenin and $\mathrm{MyHC}$ expression in mouse differentiating myotubes. Myogenin+ nuclei ratio and fusion index were quantified. $n=5$ independent wells. (D) Expression of $B m p 3 b$ in mesenchymal progenitors isolated from intact or CTX-injured muscle. $n=4$. (E) $\mathrm{MyHC}^{+}$area and fusion index of human myotubes with or without BMP3B treatment. $n$ $=11$ randomly selected fields. (F) Phosphorylation of Smad2, Smad1/$5 /-8$, and Akt in human myotubes was examined. Data of cells derived from 2 different subjects are shown. Myostatin (MSTN) and BMP4 were used as positive controls. (G) Phosphorylation of Smad2, Smad1/-5/-8, and Akt in TA muscle was examined. GAPDH was used as an internal control. Data represent the mean \pm SD; 2 -sided unpaired $t$ test $(\mathbf{C}-\mathbf{E})$, ANOVA followed by Tukey's test (A and $\mathbf{B}$ ). ${ }^{*} P<0.01,{ }^{*} P<0.05$ ( $\mathbf{A}$ and B). Scale bars: $75 \mu \mathrm{m}(\mathbf{A}-\mathbf{C})$.

Thus, mesenchymal progenitor populations in certain tissues may be specialized to suitably sustain the parenchyma of that tissue. Although we revealed the indispensability of muscle-resident mesenchymal progenitors, exploring specific roles for mesenchymal populations in supporting the parenchyma of other tissues will facilitate the understanding of the mechanisms involved in the homeostasis and aging of diverse organs.

\section{Methods}

\section{Mice}

C57BL/6 mice and GFP-Tg mice were purchased from Japan SLC. Pdgfra-CreER mice (19) (stock 018280), R26-EYFP mice (46) (stock 018280), and R26-DTA mice (47) (stock 010527) were purchased from The Jackson Laboratory. Bmp3b/Gdf1O-KO mice (26) were provided by Se-Jin Lee (Jackson Laboratory for Genomic Medicine, Farmington, Connecticut, USA). CAG-CAT-mBmp3b-Tg mice have previously been described (27). Genetically engineered mice were backcrossed to C57BL/6 mice at least 6 times. Tmx (4 $\mathrm{mg}$, MilliporeSigma) was injected intraperitoneally for 5 consecutive days at 10-11 weeks of age to induce recombination. Thirteen-week-old male $B m p 3 b / G d f 1 O-K O$ and WT littermate mice were used, while female mice were used in other experiments. We used 8- to 12-week-old C57BL/6N females as young mice and 24to 28 -month-old $\mathrm{C} 57 \mathrm{BL} / 6 \mathrm{~N}$ females as aged mice.

\section{Human muscle samples}

Muscle samples were obtained from 6 subjects with an age range of 30 to 48 years for the young-middle-aged group, and 7 subjects with an age range of 73 to 90 years for the aged group. In the young-middle-aged group, 4 samples were obtained from the gluteus medius and 2 from the vastus medialis. In the aged group, 4 samples were obtained from the gluteus medius and another 3 samples were obtained from the TA.

\section{Cell isolation}

Skeletal muscle. Cell isolation was performed as described previously (48). Minced muscles were digested with $0.2 \%$ type II collagenase (Worthington) for 60 minutes (30 minutes for human muscles) at $37^{\circ} \mathrm{C}$. Digested muscles were passed through an $18-\mathrm{G}$ needle several times and further digested for 15 minutes at $37^{\circ} \mathrm{C}$. Muscle slurries were filtered through a $100 \mu \mathrm{m}$ cell strainer and then through a $40 \mu \mathrm{m}$ cell strainer (BD Biosciences). Mouse cells were resuspended in a washing buffer consisting of $2.5 \%$ FBS in PBS and stained for cell sorting. Human cells were cultured on a collagen I-coated dish (Iwaki) in growth medium (GM) consisting of DMEM supplemented with $20 \%$ FBS, $1 \%$ penicillin-streptomycin, and 2.5 $\mathrm{ng} / \mathrm{mL}$ bFGF (Katayama Chemical) at $37^{\circ} \mathrm{C}$ under $5 \% \mathrm{CO}_{2}$ and $3 \% \mathrm{O}_{2}$ for several days. When cells reached $80 \%$ confluence they were trypsinized and stained with primary antibodies followed by secondary staining.

Figure 8. BMP3B stabilizes Schwann cell characteristics. (A) Expression of Schwann cell-related genes in rat primary Schwann cells with or without BMP3B stimulation. $n=6$ independent cultures. (B) In vitro myelination assay of mouse dorsal root ganglion. Myelination was visualized by MBP immunostaining. $n=5$ randomly chosen fields from more than 3 explants. Data represent the mean $\pm \mathrm{SD}$; 2 -sided unpaired $t$ test $(\mathbf{A})$, ANOVA followed by Tukey's test (B). Scale bar: $50 \mu \mathrm{m}$ (B).
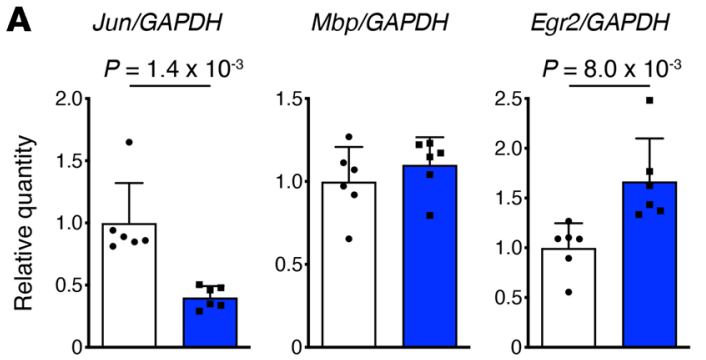

$\square$ Cont

BMP3B
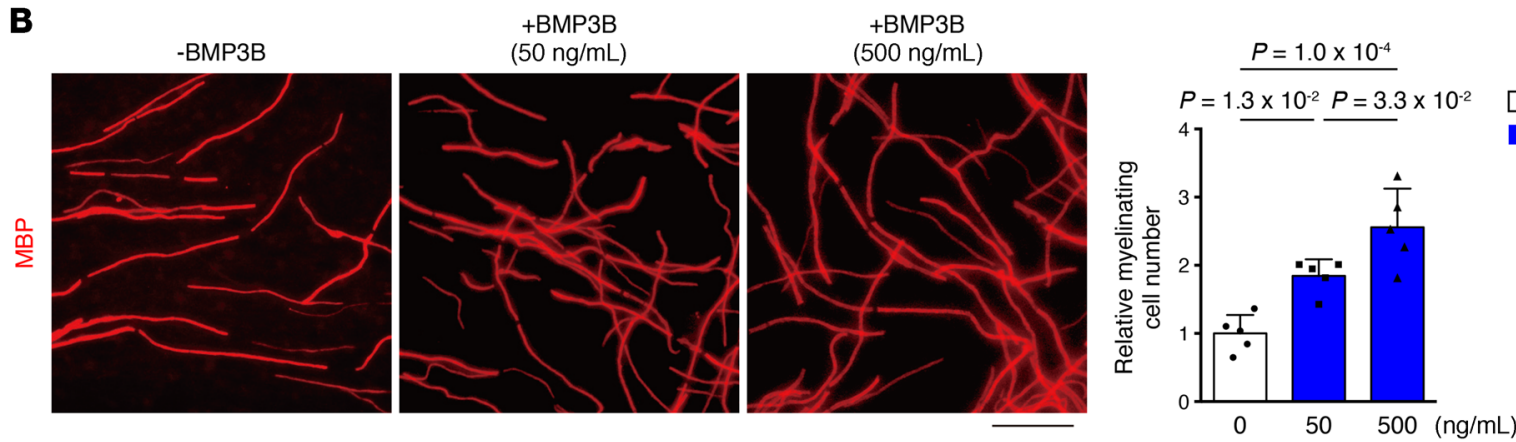


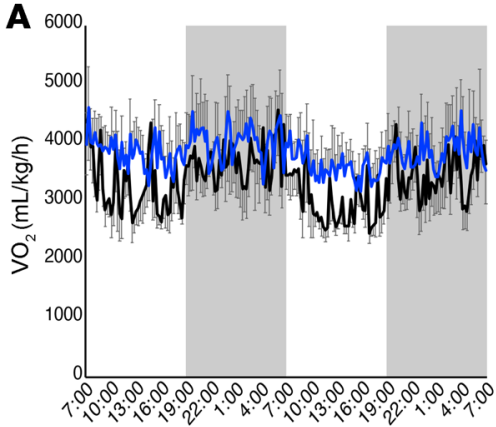

C

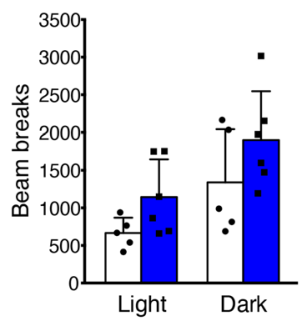

F TA EDL

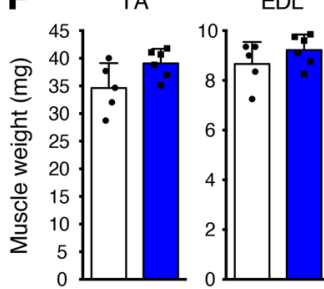

DL

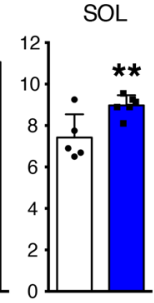

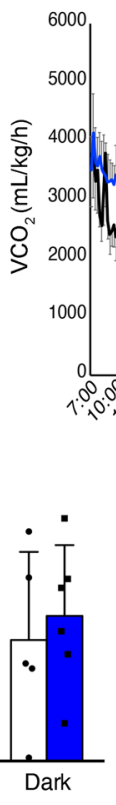
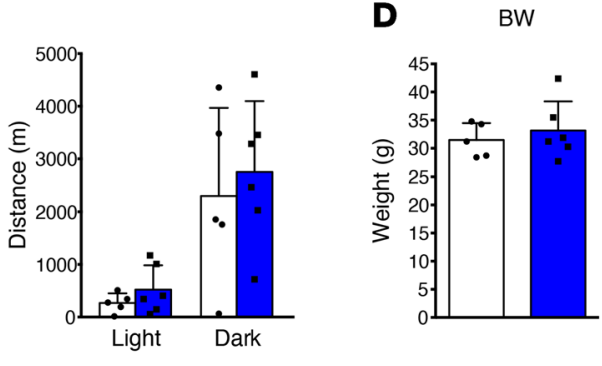

E

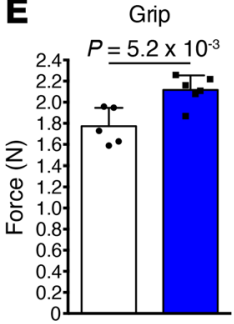

B

- Cont

- BMP3B
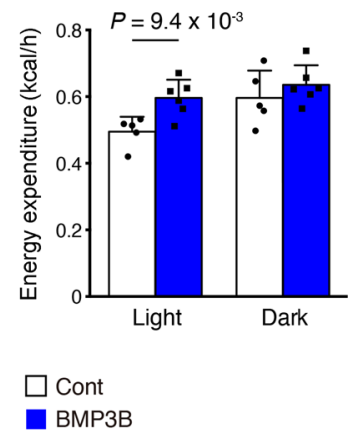

Figure 9. BMP3B administration reverses some of the sarcopenic phenotypes of aged mice. (A) Aged mice (26 months) with or without BMP3B treatment were monitored in a metabolic chamber, and oxygen consumption $\left(\mathrm{VO}_{2}\right)$ and carbon dioxide production $\left(\mathrm{VCO}_{2}\right)$ were measured. (B and C) Energy expenditure (B), and cumulative home-cage activity (left) and wheel running activity (right) (C) of BMP3B-treated or control mice. (D-F) Body weight (D), grip strength (E), and muscle weight (F) were measured. (C) The number of myofibers, CSA, and CSA distribution of RF in BMP3B-treated or control mice. $n=5$ for control, $n=6$ for BMP3B-treated mice. Data represent the mean \pm SEM (A) or the mean \pm SD (B-C); 2-sided unpaired $t$ test $(\mathbf{B}-\mathbf{C}) .{ }^{*} P<0.01,{ }^{* *} P<0.05$ (F and $\left.\mathbf{C}\right)$.

Heart. The atrium was removed, and each ventricle was finely minced. Minced hearts were digested with $0.2 \%$ type II collagenase (Worthington) for 5 minutes at $37^{\circ} \mathrm{C}$. Digested hearts were passed through an $18-\mathrm{G}$ needle several times. Digestion and needle homogenization were repeated 3 times. Slurries were filtered through a $100 \mu \mathrm{m}$ cell strainer and then through a $40 \mu \mathrm{m}$ cell strainer (BD Biosciences). Cells were resuspended in washing buffer and stained for cell sorting.

Bone marrow. Bone marrow cells were flushed out from the femurs and tibias and pooled in sterile tubes. The remaining femurs and tibias were cut into small pieces. Bone fragments were digested with $0.2 \%$ type II collagenase (Worthington) with gentle agitation for 2 hours at $37^{\circ} \mathrm{C}$. Digested bone fragments were filtered through a $100 \mu \mathrm{m}$ cell strainer and then through a $40 \mu \mathrm{m}$ cell strainer (BD Biosciences). Filtered cells were mixed with pooled bone marrow cells. Cells were resuspended in washing buffer and stained for cell sorting.

Reagents used for cell surface staining are listed in Supplemental Table 2. Isotype control staining is presented in Supplemental Figure 6. Cell sorting was performed with the MoFlo Astrios (Beckman Coulter) or SORP FACSAria II (BD Biosciences) instrument.

\section{EBD injection}

EBD (MillipreSigma) was dissolved in PBS at a concentration of $1 \%$. One day prior to the analysis, EBD $(10 \mu \mathrm{L} / \mathrm{g})$ was injected intraperitoneally to detect damaged myofibers. Damaged myofibers were visualized by histological analysis of the intracellular accumulation of EBD and intracellular staining of mouse IgG (20).

\section{Immunohistochemistry, cytochemistry, and microscopy}

Fresh muscle samples were rapidly frozen in isopentane cooled with liquid nitrogen. For the detection of EYFP or GFP and the observation of NMJs, muscles were fixed in 4\% paraformaldehyde (PFA) for 30 minutes, immersed sequentially in $10 \%$ and $20 \%$ sucrose, and frozen. For the observation of NMJ, $40 \mu \mathrm{m}$ thick longitudinal sections were obtained using a cryostat and treated with $1 \mu \mathrm{g} / \mathrm{mL} \alpha$-bungarotoxin ( $\alpha$ BTX) (Invitrogen) for 1 hour. After washing with PBS, the sections were treated with methanol for 5 minutes at $-20^{\circ} \mathrm{C}$. In other cases, 7 $\mu \mathrm{m}$ thick transverse sections were obtained. Fresh frozen sections were fixed with $4 \%$ PFA for 5 minutes. For the staining of myosin heavy chain (MyHC), fresh frozen sections were fixed with acetone for 5 minutes at $-20^{\circ} \mathrm{C}$. Cultured cells were fixed with $4 \%$ PFA for 5 minutes. Specimens were blocked with protein-block serum-free reagent (DAKO) for 
15 minutes and incubated with primary antibodies at $4^{\circ} \mathrm{C}$ overnight, followed by secondary staining. Antibodies used are listed in Supplemental Table 2. Specimens were counterstained with DAPI (Invitrogen) and mounted with SlowFade Gold anti-fade reagent (Invitrogen). Images were captured using an inverted fluorescence microscope DMI6000B (Leica), BZ-X710 (Keyence), confocal laser scanning microscope system LSM700 (Carl Zeiss), or TCS SP8 (Leica). Negative immunostaining controls are presented in Supplemental Figure 6.

\section{Quantitative analysis of myofibers}

Cross sections were obtained by cutting at the mid-belly of muscle (approximately $3 \mathrm{~mm}$ from the proximal end of the TA muscle or approximately $4 \mathrm{~mm}$ from the distal end of the quadriceps femoris muscle). Immunostaining of laminin or dystrophin was performed, and fluorescence images of entire cross sections were captured with a BZ-X710 fluorescence microscope system. Image recognition and quantification were performed using the Hybrid Cell Count Application (Keyence). For RF measurement, the region of interest (ROI) was manually set to RF. Laminin-positive basal lamina or dystrophin-positive sarcolemma was first recognized based on the intensity of the fluorescence signal by adjusting the threshold, and myofibers were recognized using an inversion function. The separation function was used to efficiently separate individual myofibers. The small, misrecognized areas were excluded by adjusting the lower limit in the histogram function. Finally, errors in the recognition step were corrected manually. The myofiber number and CSA of individual myofibers were calculated subsequently.

\section{Reconstitution of mesenchymal progenitor cells}

$P \alpha-C E / R 26-D T A$ mice were injected with Tmx as described above. One day after Tmx injection, CD31-CD45-PDGFR $\alpha^{+}$mesenchymal progenitors were sorted from GFP-Tg mice, and $1 \times 10^{5}$ to $2 \times 10^{5}$ freshly sorted cells were transplanted into the TA muscles of $P \alpha-C E / R 26$ $D T A$ mice. Contralateral TA muscles were injected with PBS and used as controls. Transplantation was performed 3 times at weekly intervals. In the third injection, $1.5 \mu \mathrm{M} \mathrm{DiIC}_{18}$ (5)-DS (DiD, Invitrogen) was injected into the control TA muscles. To assess myofiber CSA, $7 \mu \mathrm{m}$ thick transverse sections were obtained at $200 \mu \mathrm{m}$ intervals throughout the muscle, and the section showing the maximal distribution of $\mathrm{GFP}^{+}$cells or DiD was chosen for analysis. The ROI was set corresponding to the $\mathrm{GFP}^{+}$cell- or DiD-distributed area, and CSA was measured using the Hybrid Cell Count Application (Keyence).

\section{Whole-mount immunofluorescence staining}

The extensor digitorum longus muscle was split into 4 pieces and fixed with $4 \%$ PFA for 30 minutes. After washing with PBS, the muscles were blocked by incubating overnight in a blocking solution consisting of $1 \%$ Triton X-100 and $4 \%$ BSA in PBS at $4^{\circ} \mathrm{C}$, followed by incubation for 1 day with primary antibodies diluted with the blocking solution at $4^{\circ} \mathrm{C}$ with rotation. Subsequently, the muscles were incubated for 1 day with secondary reagents diluted with the blocking solution at $4^{\circ} \mathrm{C}$ with rotation. Stained muscles were counterstained with DAPI (Invitrogen) and mounted with SlowFade Gold anti-fade reagent (Invitrogen). $Z$-stack images were captured using the LSM 700 confocal laser scanning microscope system (Carl Zeiss), and reconstructed images were displayed by shadow projection using ZEN software (Carl Zeiss).

\section{Assessment of innervation at the NMJs}

$Z$-stack images of $40 \mu \mathrm{m}$ thick longitudinal TA sections were captured using the TCS SP8 confocal laser scanning microscope system (Leica), and reconstructed images were displayed by maximum intensity projection using LAS X software (Leica). For denervation assessment, we analyzed 50-100 NMJs per TA muscle. The numbers of completely denervated, partially denervated, and innervated NMJs were counted.

\section{RNA extraction and quantitative PCR}

Total RNA was extracted from muscle tissues, sorted cells, or cultured cells by using the miRNeasy Mini Kit or RNeasy Micro Kit (Qiagen), and equal amounts of RNA were reverse transcribed into cDNA using the QuantiTect Reverse Transcription Kit (Qiagen). Real-time quantitative PCR was performed with a Thermal Cycler Dice Real-Time System (TaKaRa) with SYBR Premix Ex Taq II (TaKaRa) under the following cycling conditions: $94^{\circ} \mathrm{C}$ for 30 seconds followed by 40 cycles of amplification $\left(94^{\circ} \mathrm{C}\right.$ for 5 seconds, $60^{\circ} \mathrm{C}$ for 20 seconds, $72^{\circ} \mathrm{C}$ for 10 seconds) and dissociation-curve analysis. The following genes were shown to be suitable for each experimental condition as internal control genes and used for gene expression quantification: cytidine monophospho-N-acetylneuraminic acid synthetase (Cmas) for mouse tissue $(49,50)$, NADH:ubiquinone oxidoreductase subunit A13 (NDUFA13) for human samples (51), heat shock factor binding protein 1 (Hsbp1) for mouse cells (52), and Gapdh for rat cells. Specific primer sequences used for PCR are listed in Supplemental Table 3.

\section{Microarray analysis}

Microarray analysis was performed using the 3D-Gene Mouse Oligo Chip 24K (Toray) with the following samples: gastrocnemius muscle derived from $P \alpha-C E / R 26-D T A$ mice or WT/R26-DTA mice, freshly isolated mesenchymal progenitors, satellite cells, and mixed populations of endothelial and hematopoietic cells obtained from young or aged mice. We searched for genes that were downregulated $(<0.5-$ fold) in Po-CE/R26-DTA compared with those in WT/R26-DTA muscle. We further narrowed the candidate pool by searching for genes that were preferentially expressed ( $>4$-fold) in young mesenchymal progenitors compared with those in young satellite cells or young endothelial/hematopoietic cells. Finally, we searched for genes that were downregulated $(<0.5$-fold) in aged mesenchymal progenitors compared with those in their young counterparts. The microarray data were deposited in the NCBI's Gene Expression Omnibus database with accession numbers GSE126665 and GSE126666.

\section{Cell culture}

Adipogenic differentiation. Freshly sorted CD31-CD45-PDGFR $\alpha^{+}$mesenchymal progenitors were cultured on Matrigel-coated (BD Biosciences) 48-well plates in $\mathrm{GM}$ at $37^{\circ} \mathrm{C}$ under $5 \% \mathrm{CO}_{2}$ and $3 \% \mathrm{O}_{2}$ for 4 days. For adipogenic differentiation, cells were cultured for 3 days in an adipogenic induction medium consisting of DMEM supplemented with 10\% FBS, 0.5 mM IBMX (MilliporeSigma), $0.25 \mu \mathrm{M}$ dexamethasone (MilliporeSigma), and $10 \mu \mathrm{g} / \mathrm{mL}$ insulin (MilliporeSigma), followed by adipogenic maintenance medium consisting of DMEM supplemented with $10 \% \mathrm{FBS}$ and $10 \mu \mathrm{g} / \mathrm{mL}$ insulin for 3 days at $37^{\circ} \mathrm{C}$ under $5 \% \mathrm{CO}_{2}$ and $20 \% \mathrm{O}_{2}$.

Coculture experiment. $\mathrm{CD} 31^{-} \mathrm{CD} 45^{-} \mathrm{SM} / \mathrm{C}-2.6^{+}$satellite cells were sorted from WT mice and CD31-CD 45-PDGFR $\alpha^{+}$mesenchymal progenitors were sorted from $\mathrm{P} \alpha / \mathrm{Bmp} 3 b$-Tg or $\mathrm{Bmp} 3 b$-KO mice. For sin- 
gle culture, $2 \times 10^{3}$ (single) or $6 \times 10^{3}($ single $\times 3$ ) satellite cells were cultured per well in a 96-well CellCarrier Ultra plate (PerkinElmer). For coculture, $2 \times 10^{3}$ satellite cells and $4 \times 10^{3}$ mesenchymal progenitors were plated per well. Cells were maintained in $\mathrm{GM}$ at $37^{\circ} \mathrm{C}$ under $5 \% \mathrm{CO}_{2}$ and $3 \% \mathrm{O}_{2}$ for 4 days and treated with differentiation medium (DM) consisting of DMEM supplemented with $5 \%$ horse serum for 2 days at $37^{\circ} \mathrm{C}$ under $5 \% \mathrm{CO}_{2}$ and $20 \% \mathrm{O}_{2}$. The medium was replaced with fresh DM, and cells were maintained for an additional 2 days. To analyze the myotube area, cells were fixed with 4\% PFA for 5 minutes followed by immunofluorescence staining of MyHC. For quantification, 6 randomly selected fields from duplicate wells were used. Images were collected and pooled from 2 independent experiments. The area of the myotube in 12 randomly selected fields was measured collectively using the WinROOF software (Mitani Corp).

Conditioned culture. The effects of $\mathrm{CM}$ or $\mathrm{BMP} 3 \mathrm{~B}$ on mature human myotubes were examined by modifying a previously published method (53). Mesenchymal progenitors sorted from $P \alpha / B m p 3 b$-Tg or $\mathrm{Bmp} 3 \mathrm{~b}$ - $\mathrm{KO}$ mice were cultured in $\mathrm{GM}$ at $37^{\circ} \mathrm{C}$ under $5 \% \mathrm{CO}_{2}$ and $3 \%$ $\mathrm{O}_{2}$ for 5 days. The medium was replaced with a serum-free medium consisting of DMEM with 2\% serum replacement 1 reagent (MilliporeSigma) for 1 day. The supernatant was collected and used as mesenchymal progenitor CM. CD56 ${ }^{+} \mathrm{CD} 82^{+}$PDGFR $\alpha^{-} \mathrm{CD} 201^{-}$human myoblasts were prepared as described previously (54). Human myoblasts were cultured in 96-well CellCarrier Ultra plates (PerkinElmer) in $\mathrm{GM}$ at $37^{\circ} \mathrm{C}$ under $5 \% \mathrm{CO}_{2}$ and $3 \% \mathrm{O}_{2}$ and induced to differentiate into myotubes by culturing in $\mathrm{DM}$ at $37^{\circ} \mathrm{C}$ under $5 \% \mathrm{CO}_{2}$ and $20 \% \mathrm{O}_{2}$ for 5 days. Differentiated myotubes were then maintained in GM for 2 days. Myotubes were serum-starved by maintenance in serum-free medium for 4 hours and stimulation with CM. To assess the phosphorylation of signaling molecules, myotubes were lysed in lysis buffer for 30 minutes following stimulation with $\mathrm{CM}$. To analyze the myotube area, myotubes were maintained in $\mathrm{CM}$ for 3 days and the area of the myotube was measured as described above.

BMP3B stimulation. Recombinant BMP3B (R\&D Systems) was dissolved in a serum-free medium. The effect of BMP3B on human myotubes was examined similarly to that of the conditioned culture. Recombinant GDF8/myostatin (R\&D Systems) and BMP4 (R\&D Systems) were used as positive controls for Smad2 and Smad1/-5/-8 signaling pathways.

Culture of primary Schwann cells derived from rats. Primary Schwann cell cultures were prepared as previously described (55) with minor modifications. Briefly, Schwann cells were obtained from the sciatic nerves of postnatal 2-day-old Sprague-Dawley rat pups. Contaminating fibroblasts were removed from the culture by treating the cells with $10 \mu \mathrm{M}$ cytosine arabinoside for 48 hours and complement-mediated cytolysis using anti-Thy1.1 (Serotec) and rabbit complement (Cappel). Schwann cells were propagated on poly-L-lysine-coated plates in DMEM supplemented with $10 \%$ FBS, $2 \mu \mathrm{M}$ forskolin, and $20 \mathrm{ng} / \mathrm{mL}$ rh-HRG- $\beta 1$ (MilliporeSigma). Schwann cells were stimulated with DMEM containing 10\% FBS and $500 \mathrm{ng} / \mathrm{mL} \mathrm{BMP3B}$ for 4 days and lysed in lysis buffer (Qiagen) for gene expression analysis.

\section{In vitro myelination assay}

In vitro myelination assays were performed as described previously (56). Murine DRG explants dissected from the E12.5 embryos of C57BL/6J mice were cultured on poly-L-lysine- and laminin-coated 24-well plates in DMEM containing 10\% FBS and $50 \mathrm{ng} / \mathrm{mL}$ nerve growth factor (Harlan Bioproducts) for 7 days. Myelination was induced by the addition of ascorbic acid. For the analysis of myelin profiles by immunocytochemistry, the culture was fixed with $4 \%$ PFA for 5 minutes 10-14 days after initiation of myelination. Myelination profiles were visualized by immunofluorescence staining of MBP. For quantification, myelination profiles in 5 randomly chosen fields from more than 3 explants were counted using a $20 \times$ objective lens, and the number of myelinated nerve fibers per arbitrary unit area was calculated.

\section{Immunoblotting and capillary-based immunoassays}

Cells were lysed in a lysis buffer consisting of $10 \mathrm{mM}$ Tris- $\mathrm{HCl}(\mathrm{pH}$ 7.5), $150 \mathrm{mM} \mathrm{NaCl}, 1 \%$ sodium deoxycholate, $1 \%$ Triton $\mathrm{X}-100$, protease inhibitor cocktail (Roche), and phosphatase inhibitor cocktail (Roche). After sonication for 5 minutes, the lysates were centrifuged at $1000 \mathrm{~g}$ for 15 minutes at $4^{\circ} \mathrm{C}$ and the supernatants were recovered. The protein concentration was determined using the Pierce $660 \mathrm{~nm}$ Protein Assay (Thermo Fisher Scientific). Aliquots of the lysates containing $10 \mu \mathrm{g}$ of protein were separated by sodium dodecyl sulfate-polyacrylamide gel electrophoresis and transferred onto polyvinylidene difluoride membranes. The membranes were probed with primary antibodies followed by secondary incubation with horseradish peroxidase-conjugated secondary antibodies. After the chemiluminescence reaction, images of the developed immunoblots were captured using a light-capture imaging system (ATTO). In some cases, the proteins were separated and detected using an automated capillary electrophoresis system Wes (ProteinSimple) with capillary cartridges of 12-230 $\mathrm{kDa}$ (ProteinSimple). Signals were analyzed and visualized using Compass software (ProteinSimple).

\section{Grip strength test}

The peak grip force was measured using a grip strength meter (Muromachi Kikai). Measurements were performed 5 times for each mouse, and the maximum values were recorded.

\section{BMP3B administration and metabolic experiments}

An osmotic pump (Alzet) with a $200 \mu \mathrm{L}$ reservoir volume and a 2-week release duration was filled with $75 \mu \mathrm{g}$ of recombinant BMP3B (R\&D Systems) dissolved in $4 \mathrm{mM} \mathrm{HCl}$. The control pump was filled with $4 \mathrm{mM} \mathrm{HCl}$. Filled pumps were subcutaneously transplanted into 26 -month-old C57BL/6N females. After 2 weeks, the mice were housed in a metabolic cage equipped with a running wheel (Shinfactory) for 3 days. Mice were acclimated for the first 24 hours and then monitored for 48 hours. The oxygen consumption, carbon dioxide production, and energy expenditure were measured using an Oxymax open-circuit indirect calorimetry system (Columbus Instruments). Home-cage activity and wheel-running activity were measured using the Actimo dual activity monitoring system (Shinfactory). Mice were subjected to the grip strength test prior to euthanizing.

\section{Statistics}

Statistical significance was assessed using Excel (Microsoft) and Prism 8 (GraphPad Software). No statistical methods were used to predetermine the sample size. Body weight, muscle weight, and grip strength measurements were performed in a blinded manner; other experi- 
ments were performed in a nonblinded setup. Two-tailed unpaired and paired Student's $t$ tests were used for comparisons between 2 groups. For comparisons of more than 2 groups, 1-way ANOVA followed by Dunnett's test or Tukey's post hoc test was used. $P$ values of less than 0.05 were considered statistically significant.

\section{Study approval}

Experiments using mice were approved by the Experimental Animal Care and Use Committee of Tokyo Metropolitan Geriatric Hospital and Institute of Gerontology. Experiments using human samples were approved by the Ethical Committee at the Tokyo Metropolitan Geriatric Hospital and Institute of Gerontology, and Fujita Health University. All subjects provided written informed consent.

\section{Author contributions}

AU conceived the study and was responsible for designing and performing experiments, analyzing data, and writing the manuscript. MIU helped with FACS, immunohistochemical, and metabolic experiments, and HZ, TK, and YY helped with cell culture and RNA experiments. $\mathrm{MN}, \mathrm{KH}$, and $\mathrm{H}$ Yamaguchi performed protein analysis and immunoblotting. SW and TA performed experiments involving Schwann cells. MM, H Yamada,
MT, NK, and TN performed human cell experiments. JH provided mouse models. AU, SF, and KT interpreted the results and coordinated the project.

\section{Acknowledgments}

We thank Se-Jin Lee for providing the Bmp3b/Gdf1O-KO mice, and Kenji Kangawa and Mikiya Miyazato for their helpful discussions. We thank Editage (www.editage.com) for English language editing. AU was funded by JSPS KAKENHI grant number JP19H04063, an AMED Practical Research Project for Rare/Intractable Diseases (number 17ek0109174h9903), an Intramural Research Grant for Neurological and Psychiatric Disorders of NCNP (number 28-6), the Japan Foundation for Aging And Health, The Naito Foundation, The General Insurance Association of Japan, Research Fund of Mitsukoshi Health and Welfare Foundation, Astellas Foundation for Research on Metabolic Disorders, Research Fund of Mitsui Sumitomo Insurance Welfare Foundation, and ONO Medical Research Foundation.

Address correspondence to: Akiyoshi Uezumi, Muscle Aging and Regenerative Medicine, Tokyo Metropolitan Institute of Gerontology (TMIG), 35-2 Sakae-cho, Itabashi, Tokyo 173-0015, Japan. Phone: 81.3.3964.3241; Email: uezumi@tmig.or.jp.
1. Goodpaster BH, et al. The loss of skeletal muscle strength, mass, and quality in older adults: the health, aging and body composition study. JGerontol A Biol Sci Med Sci. 2006;61(10):1059-1064.

2. Marcus RL, Addison O, Kidde JP, Dibble LE, Lastayo PC. Skeletal muscle fat infiltration: impact of age, inactivity, and exercise. J Nutr Health Aging. 2010;14(5):362-366.

3. Addison $\mathrm{O}$, et al. Intramuscular fat and inflammation differ in older adults: the impact of frailty and inactivity. J Nutr Health Aging. 2014;18(5):532-538.

4. Marcus RL, Addison O, LaStayo PC. Intramuscular adipose tissue attenuates gains in muscle quality in older adults at high risk for falling. A brief report. $J$ Nutr Health Aging. 2013;17(3):215-218.

5. Uezumi A, Fukada S, Yamamoto N, Takeda S, Tsuchida K. Mesenchymal progenitors distinct from satellite cells contribute to ectopic fat cell formation in skeletal muscle. Nat Cell Biol. 2010;12(2):143-152.

6. Joe AW, et al. Muscle injury activates resident fibro/adipogenic progenitors that facilitate myogenesis. Nat Cell Biol. 2010;12(2):153-163.

7. Uezumi A, et al. Fibrosis and adipogenesis originate from a common mesenchymal progenitor in skeletal muscle. JCell Sci. 2011;124(pt 21):3654-3664.

8. Ito $\mathrm{T}$, et al. Imatinib attenuates severe mouse dystrophy and inhibits proliferation and fibrosis-marker expression in muscle mesenchymal progenitors. Neuromuscul Disord. 2013;23(4):349-356.

9. Wosczyna MN, Biswas AA, Cogswell CA, Goldhamer DJ. Multipotent progenitors resident in the skeletal muscle interstitium exhibit robust BMP-dependent osteogenic activity and mediate heterotopic ossification. J Bone Miner Res. 2012;27(5):1004-1017.

10. Lees-Shepard JB, et al. Activin-dependent signaling in fibro/adipogenic progenitors causes fibro- dysplasia ossificans progressiva. Nat Commun . 2018;9(1):471.

11. Lepper C, Partridge TA, Fan CM. An absolute requirement for Pax7-positive satellite cells in acute injury-induced skeletal muscle regeneration. Development. 2011;138(17):3639-3646.

12. Murphy MM, Lawson JA, Mathew SJ, Hutcheson DA, Kardon G. Satellite cells, connective tissue fibroblasts and their interactions are crucial for muscle regeneration. Development. 2011;138(17):3625-3637.

13. Sambasivan R, et al. Pax7-expressing satellite cells are indispensable for adult skeletal muscle regeneration. Development. 2011;138(17):3647-3656.

14. Wosczyna MN, et al. Mesenchymal stromal cells are required for regeneration and homeostatic maintenance of skeletal muscle. Cell Rep. 2019;27(7):2029-2035.e5.

15. Grounds MD. Therapies for sarcopenia and regeneration of old skeletal muscles: more a case of old tissue architecture than old stem cells. Bioarchitecture. 2014;4(3):81-87.

16. Grounds MD. The need to more precisely define aspects of skeletal muscle regeneration. Int J Biochem Cell Biol. 2014;56:56-65.

17. Fry CS, et al. Inducible depletion of satellite cells in adult, sedentary mice impairs muscle regenerative capacity without affecting sarcopenia. Nat Med. 2015;21(1):76-80.

18. Keefe AC, et al. Muscle stem cells contribute to myofibres in sedentary adult mice. Nat Commun 2015;6:7087.

19. Kang SH, Fukaya M, Yang JK, Rothstein JD, Bergles DE. NG2 ${ }^{+} \mathrm{CNS}$ glial progenitors remain committed to the oligodendrocyte lineage in postnatal life and following neurodegeneration. Neuron. 2010;68(4):668-681.

20. Straub V, Rafael JA, Chamberlain JS, Campbell KP. Animal models for muscular dystrophy show different patterns of sarcolemmal disruption.
JCell Biol. 1997;139(2):375-385.

21. Ciciliot S, Rossi AC, Dyar KA, Blaauw B, Schiaffino S. Muscle type and fiber type specificity in muscle wasting. Int J Biochem Cell Biol. 2013;45(10):2191-2199.

22. Chai RJ, Vukovic J, Dunlop S, Grounds MD, Shavlakadze T. Striking denervation of neuromuscular junctions without lumbar motoneuron loss in geriatric mouse muscle. PLoS One. 2011;6(12):e28090.

23. Lombardi R, et al. Cardiac fibro-adipocyte progenitors express desmosome proteins and preferentially differentiate to adipocytes upon deletion of the desmoplakin gene. Circ Res. 2016;119(1):41-54.

24. Soliman H, et al. Pathogenic potential of Hic1-expressing cardiac stromal progenitors. Cell Stem Cell. 2020;26(2):205-220.e8.

25. Morikawa S, et al. Prospective identification, isolation, and systemic transplantation of multipotent mesenchymal stem cells in murine bone marrow. JExp Med. 2009;206(11):2483-2496.

26. Zhao R, Lawler AM, Lee SJ. Characterization of GDF-10 expression patterns and null mice. Dev Biol. 1999;212(1):68-79.

27. Hino J, et al. Overexpression of bone morphogenetic protein-3b (BMP-3b) in adipose tissues protects against high-fat diet-induced obesity. Int JObes (Lond). 2017;41(4):483-488.

28. Sartori R, et al. BMP signaling controls muscle mass. Nat Genet. 2013;45(11):1309-1318.

29. Winbanks CE, et al. The bone morphogenetic protein axis is a positive regulator of skeletal muscle mass. J Cell Biol. 2013;203(2):345-357.

30. Lai KM, et al. Conditional activation of akt in adult skeletal muscle induces rapid hypertrophy. Mol Cell Biol. 2004;24(21):9295-9304.

31. Moriya N, Miyazaki M. Akt1 deficiency diminishes skeletal muscle hypertrophy by reducing satellite cell proliferation. Am J Physiol Regul Integr 
Comp Physiol. 2018;314(5):R741-R751.

32. Barik A, Li L, Sathyamurthy A, Xiong WC, Mei L. Schwann cells in neuromuscular junction formation and maintenance. J Neurosci. 2016;36(38):9770-9781.

33. Yu Y, Xiao CH, Tan LD, Wang QS, Li XQ, Feng YM. Cancer-associated fibroblasts induce epithelial-mesenchymal transition of breast cancer cells through paracrine TGF- $\beta$ signalling. $\mathrm{Br} \mathrm{J}$ Cancer. 2014;110(3):724-732.

34. Zhou T, et al. GDF10 inhibits proliferation and epithelial-mesenchymal transition in triple-negative breast cancer via upregulation of Smad7. Aging (Albany NY). 2019;11(10):3298-3314.

35. Ohkawara B, Iemura S, ten Dijke P, Ueno N. Action range of $\mathrm{BMP}$ is defined by its $\mathrm{N}$-terminal basic amino acid core. Curr Biol. 2002;12(3):205-209.

36. Muhl L, et al. Single-cell analysis uncovers fibroblast heterogeneity and criteria for fibroblast and mural cell identification and discrimination. Nat Comm. 2020;11(1):3953.

37. Li S, et al. GDF10 is a signal for axonal sprouting and functional recovery after stroke. Nat Neurosci. 2015;18(12):1737-1745.

38. Camps J, et al. Interstitial cell remodeling promotes aberrant adipogenesis in dystrophic muscles. Cell Rep. 2020;31(5):107597.

39. De Stefano F, et al. Obesity, muscular strength, muscle composition and physical performance in an elderly population. J Nutr Health Aging. 2015;19(7):785-791.

40. Lukjanenko L, et al. Aging disrupts muscle stem cell function by impairing matricellular WISP1 secretion from fibro-adipogenic progenitors. Cell Stem Cell. 2019;24(3):433-446.e7.

41. Scott RW, Arostegui M, Schweitzer R, Rossi FMV, Underhill TM. Hic1 defines quiescent mesenchymal progenitor subpopulations with distinct functions and fates in skeletal muscle regeneration. Cell Stem Cell. 2019;25(6):797-813.e9.

42. Uezumi A, Ikemoto-Uezumi M, Tsuchida K. Roles of nonmyogenic mesenchymal progenitors in pathogenesis and regeneration of skeletal muscle. Front Physiol. 2014;5:68.

43. Santini MP, et al. Tissue-resident PDGFR $\alpha^{+}$progenitor cells contribute to fibrosis versus healing in a context- and spatiotemporally dependent manner. Cell Rep. 2020;30(2):555-570.e7.

44. Omatsu $Y$, et al. The essential functions of adipo-osteogenic progenitors as the hematopoietic stem and progenitor cell niche. Immunity. 2010;33(3):387-399.

45. Omatsu Y, Seike M, Sugiyama T, Kume T, Nagasawa T. Foxc1 is a critical regulator of haematopoietic stem/progenitor cell niche formation. Nature. 2014;508(7497):536-540.

46. Srinivas S, et al. Cre reporter strains produced by targeted insertion of EYFP and ECFP into the ROSA26 locus. BMC Dev Biol. 2001;1:4.

47. Wu S, Wu Y, Capecchi MR. Motoneurons and oligodendrocytes are sequentially generated from neural stem cells but do not appear to share common lineage-restricted progenitors in vivo. Development. 2006;133(4):581-590.

48. Uezumi A, Kasai T, Tsuchida K. Identification, isolation, and characterization of mesenchymal progenitors in mouse and human skeletal muscle. Methods Mol Biol. 2016;1460:241-253.

49. Zhao P, Hoffman EP. Embryonic myogenesis pathways in muscle regeneration. Dev Dyn. 2004;229(2):380-392.

50. Yoshimoto Y, Ikemoto-Uezumi M, Hitachi K, Fukada SI, Uezumi A. Methods for accurate assessment of myofiber maturity during skeletal muscle regeneration. Front Cell Dev Biol. 2020;8:267.

51. Uezumi A, et al. Identification and characterization of PDGFR $\alpha+$ mesenchymal progenitors in human skeletal muscle. Cell Death Dis. 2014;5:e1186.

52. Ikemoto-Uezumi M, et al. Pro-insulin-like growth factor-II ameliorates age-related inefficient regenerative response by orchestrating self-reinforcement mechanism of muscle regeneration. Stem Cells. 2015;33(8):2456-2468.

53. Egerman MA, et al. GDF11 increases with age and inhibits skeletal muscle regeneration. Cell Metab. 2015;22(1):164-174.

54. Uezumi A, et al. Cell-surface protein profiling identifies distinctive markers of progenitor cells in human skeletal muscle. Stem Cell Reports. 2016;7(2):263-278.

55. Brockes JP, Fields KL, Raff MC. Studies on cultured rat Schwann cells. I. Establishment of purified populations from cultures of peripheral nerve. Brain Res. 1979;165(1):105-118.

56. Saitoh F, Wakatsuki S, Tokunaga S, Fujieda H, Araki T. Glutamate signals through mGluR2 to control Schwann cell differentiation and proliferation. Sci Rep. 2016;6:29856. 